
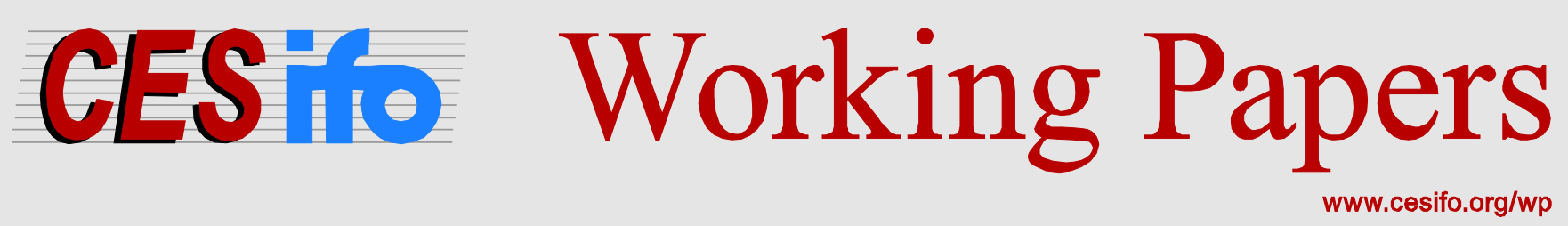

\title{
Measuring Uncertainty of a Combined Forecast and Some Tests for Forecaster Heterogeneity
}

\author{
Kajal Lahiri \\ Huaming Peng \\ Xuguang Sheng
}

\author{
CESIFO WORKING PAPER NO. 5468 \\ CATEGORY 12: EMPIRICAL AND THEORETICAL METHODS \\ August 2015
}

An electronic version of the paper may be downloaded

- from the SSRN website:

- from the RePEc website:

- from the CESifo website:

WwW.SSRN.com

Www.RePEc.org

www.CESifo-group.org/wp

ISSN 2364-1428

CESifo 


\title{
Measuring Uncertainty of a Combined Forecast and Some Tests for Forecaster Heterogeneity
}

\begin{abstract}
We have argued that from the standpoint of a policy maker, the uncertainty of using the average forecast is not the variance of the average, but rather the average of the variances of the individual forecasts that incorporate idiosyncratic risks. With a slight reformulation of the loss function and a standard factor decomposition of a panel of forecasts, we show that the uncertainty of the average forecast can be expressed as the disagreement among the forecasters plus the volatility of the common shock. Using new statistics to test for the homogeneity of idiosyncratic errors under the joint limits with both $T$ and $n$ approaching infinity simultaneously, we show that some previously used measures significantly underestimate the conceptually correct benchmark forecast uncertainty.
\end{abstract}

JEL-Code: C120, C330, E370.

Keywords: forecast combination, forecast uncertainty, model averaging, panel data.

Kajal Lahiri

Department of Economics

University at Albany, SUNY

1400 Washington Avenue

USA - 12222 Albany NY

klahiri@albany.edu

Huaming Peng

Department of Economics

University at Albany, SUNY

1400 Washington Avenue

USA - 12222 Albany NY

hpeng@albany.edu
Xuguang Sheng

Department of Economics

American University

4400 Massachusetts Avenue, NW

USA - Washington, DC 20016

sheng@american.edu 


\section{Introduction}

Consider the problem of a macro policy maker who often has to aggregate a number of competing forecasts from experts for the purpose of a uniform policy making. A general solution was provided by Bates and Granger (1969) who have inspired extensive research on forecast combination, as evidenced by the two comprehensive surveys in Clemen (1989) and Timmermann (2006), followed by many additional papers since 2006. The solution based on minimizing the mean square error of the combined forecasts calls for a performance-based weighted average of individual forecasts with precision of the combined forecast that is readily shown to be better than any of the constituent elements under reasonable conditions. We show that a slight reformulation of the Bates-Granger loss function justifies one to take the average of the individual forecast variances rather than the variance of the average as the correct measure for aggregate uncertainty. A more fundamental justification for incorporating disagreement as part of aggregate uncertainty comes from the rich literature on model averaging pioneered by Leamer (1978). ${ }^{1}$ With disagreement as a component of forecast uncertainty, the presumed superiority of the consensus forecast over the typical forecaster will be lost, and the primary advantage of averaging will be an insurance value against idiosyncratic errors that may be generated due to heterogeneous learning in periods of structural instability, breaks and bad judgements, cf. Hendry and Clements (2002). The objective of the combination approach will then be less ambitious, and will be similar to that in the machine learning literature where forecasts are combined on line to adapt to a few superior ones, see Yang (2004) and Sancetta (2010). Our study is aimed at developing a theoretically sound measure of uncertainty for the consensus forecast based on past forecast errors and is closely related to Clements (2014) that analyzed the relationship between the

\footnotetext{
${ }^{1}$ Draper (1995) and Buckland, et al. (1997) present cogent explications of the result using Bayesian and Frequentist approaches respectively, see also Sala-i-Martin, et al. (2004). Geweke and Amisano (2011) advocate the optimal linear pool that does not condition on one of the models being fully correctly specified.
} 
ex ante uncertainty and the ex post measure.

One motivation to explore the importance of using a theoretically sound uncertainty measure of the consensus forecast comes from the recent advances in the presentation and communication strategies by a number of central banks, pioneered by Bank of England's fan charts to report forecast uncertainty. For the credibility of forecasts in the long run, it is essential that the reported confidence bands for forecasts be properly calibrated. In the U.S., from November 2007, all Federal Open Market Committee (FOMC) members are required to provide their judgments as to whether the uncertainty attached to their projections is greater than, smaller than, or broadly similar to typical levels of forecast uncertainty in the past. In order to aid each FOMC member to report their personal uncertainty estimates, Reifschneider and Tulip (2007) have proposed a measure for gauging the average magnitude of historical uncertainty using information on past forecast errors from a number of private and government forecasters. These benchmark estimates for a number of target variables are reported in the minutes of each FOMC meeting and are used by the public to interpret the responses of the FOMC participants.

Another motivation comes from the recent studies on the impact of uncertainty on real economic activities following the seminal work of Bloom (2009). So far, the empirical literature has used various proxies for macro uncertainty, such as stock market volatility, disagreement among forecasters, forecast surprises and newspaper coverage of economic uncertainty. Jurado, et al. (2015) have pointed out that while all of these measures are directly observable, none of them individually are adequate as proxies for uncertainty. Lahiri and Sheng (2010) show that disagreement is a part of ex ante uncertainty and the difference between them is the perceived volatility of shocks over forecast horizons. Baker, et al. (2013) use disagreement among professional forecasters in predicting inflation and government expenditures as one component to construct indices of uncertainty about policy-related macroeconomic variables. Given the role of disagreement in constructing ex ante measures of 
uncertainty, a careful examination of its importance in measuring ex post uncertainty cannot be overemphasized.

In this paper we establish the asymptotic limits for alternative measures of ex post uncertainty under the joint limits with both the time series $(T)$ and cross section $(n)$ dimensions approaching infinity simultaneously, and develop two tests to check if the alternative uncertainty measures are statistically different. A Monte Carlo study confirms that the tests perform well in our context. We use individual inflation and output growth forecasts from the Survey of Professional Forecasters (SPF) over 1992-2013 to show that the uncertainty measure conventionally attached to a consensus forecast using the Bates-Granger approach and the Reifschneider-Tulip benchmark measure underestimate the true uncertainty, often substantially. Our tests also confirm these results at the $5 \%$ level for most of forecast horiZons.

The plan of the paper is as follows. Section 2 studies the relationship between disagreement and overall forecast uncertainty. Section 3 examines various measures of historical uncertainty, derives their respective asymptotic limits and proposes some simple statistical tests for the equivalence of these measures. In Section 4, we illustrate the underestimation of uncertainty by calculating the benchmark uncertainty using SPF data on real GDP and inflation forecasts. Finally, Section 5 summarizes the results and presents some concluding remarks. Technical proofs are relegated to an appendix.

A word on notation. $\|\cdot\|$ denotes the Euclidean norm. $\longrightarrow_{p}$ denotes convergence in probability. $\Longrightarrow$ denotes convergence in distribution. The limits taken under $(n, T \rightarrow \infty)_{\text {seq }}$ refer to sequential limits where $T$ goes to infinity first and then $n$ goes to infinity. The limits taken under $(n, T \rightarrow \infty)$ are regarded as the joint limits with both $T$ and $n$ approaching infinity at the same time. 


\section{Uncertainty and Disagreement}

\subsection{Model and Assumptions}

Let $Y_{t}$ be the random variable of interest, $F_{i t}$ be the forecast of $Y_{t}$ made by individual $i$ at time $t-h$. Then individual $i$ 's forecast error, $e_{i t}$, can be defined as

$$
e_{i t}=A_{t}-F_{i t}
$$

where $A_{t}$ is the actual realization of $Y_{t}$. Following a long tradition, e.g., Palm and Zellner (1992) and Davies and Lahiri (1995), we write $e_{i t}$ as the sum of an individual bias, $\mu_{i t}$, a common component, $\lambda_{t}$ and idiosyncratic errors, $\varepsilon_{i t}$ :

$$
e_{i t}=\mu_{i t}+\lambda_{t}+\varepsilon_{i t}
$$

where $\mu_{i t}$ is nonrandom and time-varying, $\lambda_{t}\left(=\sum_{k=0}^{h-1} \theta_{k} \zeta_{t k}\right.$, with $\left.\theta_{0}=1, \sum_{k=0}^{h-1} \theta_{k}^{2}<\infty\right)$ represents the cumulative weighted effect of all independent shocks $\zeta_{t k}$ that occurred from $h$-period ahead to the end of target year $t$. Thus even if forecasters make "perfect" forecasts, the forecast error may still be nonzero due to shocks which are, by nature, unpredictable. Forecasters, however, do not make "perfect" forecasts even in the absence of unanticipated shocks. This "lack of perfection" is due to other factors (e.g., differences in information acquisition and processing, loss functions, interpretation, judgment, and forecasting models) specific to a given individual at a given point in time and is represented by the idiosyncratic error, $\varepsilon_{i t}$.

In order to establish the relationship between different measures of uncertainty and derive their asymptotic limits, we make the following simplifying assumptions:

\section{Assumption 1 (Bias)}


$\mu_{i t}$ is nonstochastic for all $i$ and all $t$ with $\sup _{i} \frac{1}{T} \sum_{t=1}^{T} \mu_{i t}^{4}=O\left(T^{-1} n^{-\alpha}\right)$ for some $\alpha>1$.

\section{Assumption 2 (Common Shocks)}

$\lambda_{t}$ is a stationary ergodic moving average process of order at most $h-1$ with $E \lambda_{t}=0$, $E \lambda_{t}^{2}=\sigma_{\lambda}^{2}, E\left|\lambda_{t}\right|^{4+\delta}<\infty$ for some $\delta>0$, and $\operatorname{var}\left(\frac{1}{\sqrt{T}} \sum_{t=1}^{T} \lambda_{t}^{2}\right) \rightarrow \Sigma_{\lambda}>0$ as $T \rightarrow \infty$.

\section{Assumption 3 (Idiosyncratic Shocks)}

$\varepsilon_{i t}$ is independently identically distributed over $t$, and independently potentially nonidentically distributed across $i$ with $E \varepsilon_{i t}=0, E \varepsilon_{i t}^{2}=\sigma_{\varepsilon i}^{2}$ with $0<\inf _{i} \sigma_{\varepsilon i}^{2} \leq \sup _{i} \sigma_{\varepsilon i}^{2}<\infty$, $E \varepsilon_{i t}^{3}=0, \operatorname{var}\left(\varepsilon_{i t}^{2}\right)=\omega_{\varepsilon i}, 0<\inf _{i} \omega_{\varepsilon i} \leq \sup _{i} \omega_{\varepsilon i}<\infty$, and $E \varepsilon_{i t}^{8}<\infty$.

\section{Assumption 4 (Relations)}

$\lambda_{t}$ is independent of $\varepsilon_{i s}$ for all $i, t$ and $s$.

Remark 1. Assumption 1 allows for time-varying nonrandom bias, which is more general than the time-invariant assumption made in the literature and hence potentially has a wider range of applications. The condition $\sup _{i} \frac{1}{T} \sum_{t=1}^{T} \mu_{i t}^{4}=O\left(T^{-1} n^{-\alpha}\right)$ for some $\alpha>1$ helps to ensure that individual bias is negligible in the asymptotic limits involving various ex post measures of forecast uncertainty. The eventually vanishing bias condition is in line with the spillover effect that the bias gets smaller as more forecasters learn from each other, and consistent with the empirical evidence that forecasters' biases diminish over time as they gain experience, cf. Lahiri and Sheng (2008). ${ }^{2}$ By contrast, Issler and Lima (2009), in attempt to reduce the size of the forecast bias, assume a stochastic (across forecasters) time-invariant bias for each forecaster. Assumption 2 is almost identical to the Assumption 3 in Issler and Lima (2009) except for the higher moment condition, which, together with the higher moment assumption of $\varepsilon_{i t}$, is required to establish the limit theorems. The common shock $\lambda_{t}$, which is the result of a cumulation of shocks to $A_{t}$ that occurred between $t-h+1$ and $t$, follows a moving average process. This expression is due in part to the wold decomposition of the forecast series and in part to the fact that the series can be written as the sum of

\footnotetext{
${ }^{2}$ Note that the bias condition allows for heterogenous rates of individual biases approaching zero.
} 
conditional mean forecast and an unforecastable component as explained in Issler and Lima (2009). Assumption 3 is standard in errors component or factor analysis. It can also be readily extended to allow for some weak time dependence at the expense of some technical complication. The independence of $\lambda_{t}$ and $\varepsilon_{i s}$ in Assumption 4 is also common in errors component or factor studies. It helps the identification of the variance of the aggregate shock from that of the idiosyncratic errors.

Taken together, the assumptions 1-4 imply that the individual forecast error is not only an asymptotic stationary and ergodic process for any given horizon, but also has a factor structure interpretation. Given a panel of forecasts, we can decompose the average squared individual forecast errors as

$$
\frac{1}{n} \sum_{i=1}^{n} e_{i t}^{2}=\left(A_{t}-F_{\cdot t}\right)^{2}+\frac{1}{n} \sum_{i=1}^{n}\left(F_{i t}-F_{\cdot t}\right)^{2}
$$

where $F_{\cdot t}=\frac{1}{n} \sum_{j=1}^{n} F_{j t}$. Now taking time average on both sides of (3) we get a measure of historical forecast uncertainty based on past errors

$$
\frac{1}{n T} \sum_{t=1}^{T} \sum_{i=1}^{n} e_{i t}^{2}=\frac{1}{T} \sum_{t=1}^{T}\left(A_{t}-F_{\cdot t}\right)^{2}+\frac{1}{n T} \sum_{t=1}^{T} \sum_{i=1}^{n}\left(F_{i t}-F_{\cdot t}\right)^{2} .
$$

This is our suggested measure of historical uncertainty of the average forecast, and is readily seen to be the average of the individual variances observed over the sample period. Equation (4) states that the measure can be decomposed into two components: uncertainty that is common to all forecasters and uncertainty that arises from heterogeneity of individual forecasters. The first component is the empirical variance of the average that is conventionally taken as the uncertainty of the consensus forecast. It is important to note that the superior performance of the consensus forecast relative to individual forecasts follows directly from Jensen's inequality, which states that with convex loss functions, the loss associated with the 
mean forecast is generally less than the mean loss of individual forecasts, see McNees (1992) and Manski (2011). Thus, the consensus forecast is not more accurate than every individual forecast in the pool; rather it outperforms an individual forecast drawn randomly from the available forecasts. ${ }^{3}$ The second component is the disagreement among forecasters. Geweke and Amisano (2014) presented a parallel decomposition of predictive variance from Bayesian model averaging in terms of intrinsic and extrinsic variances. A similar decomposition of forecast uncertainty was also obtained by Lahiri, et al. (1988) and Wallis (2005) in cases where probability distribution of forecasts are available. While analyzing individual density forecasts reported in SPF, Zarnowitz and Lambros (1987) used the average of individual variances as the uncertainty of the average forecast.

By virtue of the assumptions 1-4, the population analog of equation (4) can be obtained as

$$
\frac{1}{n} \sum_{i=1}^{n} \sum_{t=1}^{T} E\left(e_{i t}^{2}\right)=\sigma_{\lambda}^{2}+\frac{1}{n} \sum_{i=1}^{n} \sigma_{\varepsilon i}^{2}+\frac{1}{n T} \sum_{i=1}^{n} \sum_{t=1}^{T} \mu_{i t}^{2} .
$$

It is now obvious from equation (5) that the uncertainty of the average arises from the variance of the aggregate shock common to all forecasters and from the heterogeneity of individual forecasters that contains both the average idiosyncratic variance and the average of the variance of individual biases. Recognizing that each point forecast is invariably an outcome from an underlying forecast distribution, the combined forecast should be treated conceptually as the mean of a finite mixture distribution of unobserved densities, which suggests that the variance of the combined forecast will be the average of the individual variances plus an disagreement term due to systematic biases in the individual forecasts, cf. Draper (1995) and Wallis (2005). What is not readily recognized in the literature is that apart from the disagreement coming from time-varying systematic biases (i.e., $\frac{1}{n T} \sum_{i=1}^{n} \sum_{t=1}^{T} \mu_{i t}^{2}$ ), the average of the individual variances also contains a disagreement component coming from

\footnotetext{
${ }^{3}$ Roughly one-third of individual forecasts have been found to be more accurate than a consensus, but the mix of the superior forecasters change depending on the occasion, see McNees (1992).
} 
$\frac{1}{n} \sum_{i=1}^{n} \sigma_{\varepsilon i}^{2}$. In the context of the empirical examples on real GDP and inflation forecasts that we report in Section 4, a model uncertainty audit reveals that the variance explained by the systematic bias component is small compared to the other two components in equation (5). A similar result on the insignificance of the bias term is also reported by Reifschneider and Tulip (2007). That is why we make the diminishing bias assumption (for all $i$ and $h$ ) in our context.

\subsection{The Policy Maker's Loss Function}

The above empirical measure of forecast uncertainty that carries the additional disagreement term can easily be justified by examining the objective function of the policy maker. As the former chair of Federal Reserve, Alan Greenspan pointed out that the monetary policy should be conducted in such a way that the associated risk or uncertainty is minimized with respect to all available forecasts. ${ }^{4}$ When squared losses are used in forming expectations, the forecast by the $i^{t h}$ forecaster is given by $F_{i t}=E\left(Y_{t} \mid I_{i, t-h}\right)$, where $I_{i, t-h}$ is the information set containing both the information available to a particular forecaster $i$ at time $t-h$, and the characteristics of that forecaster, and its associated individual risk is defined as $E\left\{\left[Y_{t}-E\left(Y_{t} \mid I_{i, t-h}\right)\right]^{2} \mid I_{i, t-h}\right\}$. Individual risk of similar form has recently been employed by Jurado, et al. (2015) to construct a measure of macroeconomic uncertainty. ${ }^{5}$ Thus, given individual risks, the problem for the policy maker can then be formulated as

$$
\min _{\omega_{i(t-h)}} \sum_{i=1}^{n} \omega_{i, t-h} E\left\{\left[Y_{t}-E\left(Y_{t} \mid I_{i, t-h}\right)\right]^{2} \mid I_{i, t-h}\right\},
$$

\footnotetext{
${ }^{4}$ See Alan Greenspan's original remark at a symposium sponsored by the Federal Reserve Bank of Kansas City on August 29, 2003.

${ }^{5}$ Jurado, et al. (2015) define individual uncertainty as the squared root of $E\left\{\left[X_{j t}-E\left(X_{j t} \mid I_{t-h}\right)\right]^{2} \mid I_{t-h}\right\}$, where $X_{j t}$ is the $j$ th variable and $I_{t-h}$ is the common information available to all economic agents, and construct a measure of macroeconomic uncertainty as a weighted average of individual uncertainties.
} 
where $\omega_{i, t-h}$ (subject to the constraints $0 \leq \omega_{i, t-h} \leq 1, \sum_{i=1}^{n} \omega_{i, t-h}=1$ ) is the weight assigned to the individual risk $E\left\{\left[Y_{t}-E\left(Y_{t} \mid I_{i, t-h}\right)\right]^{2} \mid I_{i, t-h}\right\}$ at time $t-h$.

Minimizing the weighted risk is equivalent to selecting the best individual forecaster in terms of the smallest mean squared error. However, the policy maker has neither perfect knowledge about how much information the $i^{\text {th }}$ forecaster possesses nor the exact characteristics of this particular forecaster inherits. The empirical risk of the $i^{\text {th }}$ forecaster is simply too volatile to be the sole indicator of forecast uncertainty faced by the policy maker. Furthermore, picking a particular forecaster ignores the uncertainty in forecaster selection process, implying that the risk computed based on one selected model or forecaster is underestimated; see, for example, Yang (2004) and Wei and Yang (2012). Thus, from the point of view of a policy maker, the individual risks are stochastic not only over time but also across forecasters since they are conditional on $I_{i, t-h}$. To avoid these drawbacks, a natural alternative to forecaster selection approach is the averaging method. The forecast averaging approach has the advantages by providing the policy maker with a robust overall risk and accounting for the uncertainty inherent in the forecaster selection process. Interestingly, using the average of individual loss functions has also been proposed and justified by Levin, et al. (2003) in designing a robust forecast-based monetary policy rules under model uncertainty, and by Woodford (2005) in designing the social loss function for a policy maker on the ground that it is consistent with both the rational and herding behavior of market participants.

A simple but popular way for the policy maker to obtain a robust overall risk function is to apply the equal weighting scheme, that is, setting $\omega_{i, t-h}=\frac{1}{n}$ for all $i$. This simple approach is optimal in the sense that it avoids the problem of estimating the weights based on covariance matrix, which is extremely tricky when $n$ is relatively large due to the estimation uncertainty and the curse of dimensionality, see, Issler and Lima (2009). So a robust overall risk function 
for the policy maker that follows Greenspan's suggestion would be

$$
\frac{1}{n} \sum_{i=1}^{n} E\left\{\left[Y_{t}-E\left(Y_{t} \mid I_{i, t-h}\right)\right]^{2} \mid I_{i, t-h}\right\} .
$$

But the ex ante overall risk function defined in (7) is not particularly useful for evaluation and calibration purposes. A natural ex post analog of (7) would be the following statistic,

$$
\frac{1}{n} \sum_{i=1}^{n} \frac{1}{T} \sum_{t=1}^{T}\left(A_{t}-F_{i t}\right)^{2}=\frac{1}{n T} \sum_{i=1}^{n} \sum_{t=1}^{T} e_{i t}^{2},
$$

which is the appropriate empirical measure of forecast uncertainty from the standpoint of a policy maker.

\section{Measures of Historical Uncertainty}

Based on the argument in Section 2, the historical risk faced by a policy maker while using a panel of forecasts from $n$ experts is simply

$$
R M S E_{L P S}=\sqrt{\frac{1}{n T} \sum_{t=1}^{T} \sum_{i=1}^{n} e_{i t}^{2}}
$$

On the other hand, the conventional choice as suggested by Bates and Granger (1969) is the root mean squared error (RMSE) of the average forecast

$$
R M S E_{A F}=\sqrt{\frac{1}{T} \sum_{t=1}^{T}\left(\frac{1}{n} \sum_{i=1}^{n} e_{i t}\right)^{2}} .
$$

With the stated objective of using the root mean squared errors made by a panel of forecasts to generate a benchmark estimate of historical forecast uncertainty, Reifschneider and Tulip (2007) propose the following measure 


$$
R M S E_{R T}=\frac{1}{n} \sum_{i=1}^{n} \sqrt{\frac{1}{T} \sum_{t=1}^{T} e_{i t}^{2}} .
$$

They explicitly recognized that their average RMSE is not the conventional $R M S E_{A F}$ associated with the average forecast as in equation (10), and noted "rather we average the individual RMSEs of our forecasts in order to generate a benchmark for the typical amount of uncertainty we might expect to be associated with the separate forecasts of the different members of our sample, including the FOMC"(Reifschneider and Tulip, 2007, p.14). Interestingly, under our framework of constructing an overall risk measure based on a panel of forecasts, the RT uncertainty measure can be derived as a natural ex post empirical analog of aggregate uncertainty measure proposed by Jurado, et al. (2015). Obviously, $R M S E_{R T}$ is not exactly the same as $R M S E_{L P S}$; but, nevertheless, it incorporates at least partially the disagreement as a component of uncertainty as shown in the following theorem.

Theorem 1. Suppose Assumptions 1-4 hold. Then as $(n, T \rightarrow \infty)$,

(i) $\sqrt{T}\left(R M S E_{A F}^{2}-\sigma_{\lambda}^{2}-\frac{1}{n^{2}} \sum_{i=1}^{n} \sigma_{\varepsilon i}^{2}\right) \rightarrow_{d} N\left(0, \Sigma_{\lambda}\right)$.

(ii) $\sqrt{T}\left(R M S E_{R T}^{2}-\left(\frac{1}{n} \sum_{i=1}^{n} \sqrt{\sigma_{\lambda}^{2}+\sigma_{\varepsilon i}^{2}}\right)^{2}\right) \rightarrow_{d} N\left(0, \phi \Sigma_{\lambda}\right)$, where $\phi=\left(\lim _{n \rightarrow \infty} \frac{1}{n} \sum_{i=1}^{n}\left(\sigma_{\lambda}^{2}+\sigma_{\varepsilon i}^{2}\right)^{1 / 2}\right)^{2}\left(\lim _{n \rightarrow \infty} \frac{1}{n} \sum_{i=1}^{n}\left(\sigma_{\lambda}^{2}+\sigma_{\varepsilon i}^{2}\right)^{-1 / 2}\right)^{2}$.

(iii) $\sqrt{T}\left(R M S E_{L P S}^{2}-\left(\sigma_{\lambda}^{2}+\frac{1}{n} \sum_{i=1}^{n} \sigma_{\varepsilon i}^{2}\right)\right) \rightarrow_{d} N\left(0, \Sigma_{\lambda}\right)$.

The following Corollary follows directly from Theorem 1.

Corollary 1. Suppose Assumptions 1-4 hold. Then as $(n, T \rightarrow \infty)$,

(i) $R M S E_{A F}^{2} \rightarrow_{p} \sigma_{\lambda}^{2}$.

(ii) $R M S E_{R T}^{2} \rightarrow_{p}\left(\lim _{n \rightarrow \infty} \frac{1}{n} \sum_{i=1}^{n} \sqrt{\sigma_{\lambda}^{2}+\sigma_{\varepsilon i}^{2}}\right)^{2}$. 
(iii) $R M S E_{L P S}^{2} \rightarrow_{p}\left(\sigma_{\lambda}^{2}+\sigma_{\varepsilon}^{2}\right)$, where $\sigma_{\varepsilon}^{2}=\lim \frac{1}{n} \sum_{i=1}^{n} \sigma_{\varepsilon i}^{2}$.

Remark 2. A direct consequence of Theorem 1(i) and Corollary 1(i) is that $\frac{1}{n} \sum_{i=1}^{n} \varepsilon_{i t} \rightarrow_{p} 0$ or $\frac{1}{n} \sum_{i=1}^{n} F_{i t} \rightarrow_{p} A_{t}+\lambda_{t}$ as $n \rightarrow \infty$, a result also obtained by Issler and Lima (2009) in their Proposition 3 for their bias-corrected average forecast, under the assumption that the common shock is a moving average process of order at most $(h-1)$. While their paper is aimed at obtaining the bias-corrected average forecast under the sequential limit where $T \rightarrow \infty$ followed by $n \rightarrow \infty$, our objective is to find an appropriate benchmark measure of overall forecast uncertainty among several alternatives and obtain their respective asymptotic limits under the joint limit where $T$ and $n$ approach infinity simultaneously. In this regard, $R M S E_{A F}$ ignores the uncertainty associated with the idiosyncratic shocks, especially when $n$ is large since $R M S E_{A F}^{2}=\sigma_{\lambda}^{2}+\frac{1}{n^{2}} \sum_{i=1}^{n} \sigma_{\varepsilon i}^{2}+O_{p}\left(T^{-1 / 2}\right)$ with $\frac{1}{n^{2}} \sum_{i=1}^{n} \sigma_{\varepsilon i}^{2}=O\left(\frac{1}{n}\right)$. By contrast, for $R M S E_{L P S}$, we have $R M S E_{L P S}^{2} \rightarrow_{p} \sigma_{\lambda}^{2}+\sigma_{\varepsilon}^{2}$ as $(n, T \rightarrow \infty)$.

Remark 3. Theorem 1 and Corollary 1 also demonstrate that $R M S E_{A F} \leq R M S E_{R T} \leq$ $R M S E_{L P S}$ in the limit, because $\sigma_{\varepsilon i}^{2} \geq 0$ for all $i$ and $\lim _{n \rightarrow \infty} \frac{1}{n} \sum_{i=1}^{n} \sqrt{\sigma_{\lambda}^{2}+\sigma_{\varepsilon i}^{2}} \leq\left(\sigma_{\lambda}^{2}+\sigma_{\varepsilon}^{2}\right)^{1 / 2}$ in view of Jensen's inequality. Thus it is clear that when compared to $R M S E_{L P S}, R M S E_{R T}$, though allows for disagreement to some degree, underestimates the forecast uncertainty in the presence of unequal idiosyncratic error variances, and the amount of underestimation is given by $\frac{1}{8}\left(\sigma_{\lambda}^{2}+\sigma_{\varepsilon}^{2}\right)^{-3 / 2} \operatorname{var}\left(\sigma_{\varepsilon i}^{2}\right)$, where $\operatorname{var}\left(\sigma_{\varepsilon i}^{2}\right)=\lim _{n \rightarrow \infty} \frac{1}{n} \sum_{i=1}^{n}\left(\sigma_{\varepsilon i}^{2}-\sigma_{\varepsilon}^{2}\right)^{2}$.

Remark 4. Interestingly, $R M S E_{L P S}$ and $R M S E_{A F}$ have the same asymptotic limit distribution, even though their means are dramatically different as explained in Remark 2. This is because the asymptotic limit involving the common shocks dominates that associated with the idiosyncratic shocks. In addition, $R M S E_{R T}$, as a measure of forecast uncertainty, is more volatile than both $R M S E_{L P S}$ and $R M S E_{A F}$ since $\phi \geq 1$ by virtue of Cauchy-Schwarz inequality. 
To check whether the idiosyncratic shocks contribute to the measure of forecast uncertainty for a particular data set, we first define $\widehat{\sigma}_{\lambda}^{2}=R M S E_{A F}^{2}, \widehat{\sigma}_{\varepsilon i}^{2}=\frac{1}{T} \sum_{t=1}^{T}\left(e_{i t}-\bar{e}_{\cdot t}\right)^{2}$ and $\widehat{\omega}_{\varepsilon i T}=\frac{1}{T} \sum_{t=1}^{T}\left(\left(e_{i t}-\bar{e}_{\cdot t}\right)^{2}-\frac{1}{T} \sum_{t=1}^{T}\left(e_{i t}-\bar{e}_{\cdot t}\right)^{2}\right)^{2}$ with $\bar{e}_{\cdot t}=\frac{1}{n} \sum_{i=1}^{n} e_{i t}$, and then test the equality of $R M S E_{A F}$ and $R M S E_{L P S}$ using the statistic presented in the next theorem.

Theorem 2. Suppose Assumptions 1-4 hold. Then under the null hypothesis that $\frac{1}{n} \sum_{i=1}^{n} \sigma_{\varepsilon i}^{2}=$ $o\left(T^{-1 / 2} n^{-1 / 2}\right)$,

$$
Z=\sqrt{n T} \widetilde{\omega}_{\varepsilon T}^{-1 / 2}\left\{R M S E_{L P S}^{2}-R M S E_{A F}^{2}\right\} \rightarrow_{d} N(0,1)
$$

as $(n, T \rightarrow \infty)$ and $\frac{n}{T} \rightarrow 0$, where $\widetilde{\omega}_{\varepsilon T}=\frac{1}{n} \sum_{i=1}^{n} \widetilde{\omega}_{\varepsilon i T}, \widetilde{\omega}_{\varepsilon i T}=\left(\frac{n}{n-1}\right)^{4} \widehat{\omega}_{\varepsilon i T}-\frac{1}{n}\left(\frac{n}{n-1}\right)^{2} \widehat{\sigma}_{\varepsilon i}^{2}\left(\frac{1}{n} \sum_{j \neq i}^{n} \widehat{\sigma}_{\varepsilon j}^{2}\right)$.

Remark 5. Instead of $\sigma_{\varepsilon}^{2}=0$, the null hypothesis we test is $\frac{1}{n} \sum_{i=1}^{n} \sigma_{\varepsilon i}^{2}=o\left(T^{-1 / 2} n^{-1 / 2}\right)$, which is needed to guarantee $\frac{\sqrt{n T}}{n} \sum_{i=1}^{n} \sigma_{\varepsilon i}^{2} \rightarrow 0$ as $(n, T \rightarrow \infty)$ in the test statistic. The use of $\widetilde{\omega}_{\varepsilon i T}$ (and hence $\widetilde{\omega}_{\varepsilon i}$ ) rather than $\widehat{\omega}_{\varepsilon i T}$ in the test is to reduce the asymptotic bias of the estimator of $\omega_{\varepsilon i}$ from order $O_{p}\left(\min \left\{T^{-1 / 2}, n^{-1}\right\}\right)$ to $O_{p}\left(\min \left\{T^{-1 / 2}, n^{-2}\right\}\right)$. The restriction $\frac{n}{T} \rightarrow 0$ is needed to ensure the the approximation errors in deriving the asymptotic distribution are negligible.

To examine whether $R M S E_{R T}$ and $R M S E_{L P S}$ give statistically different measures of uncertainty in the context of a particular data set, we note that

$$
R M S E_{L P S}-R M S E_{R T}=\frac{1}{8}\left(R M S E_{L P S}\right)^{-3} \frac{1}{n} \sum_{i=1}^{n}\left(\frac{1}{T} \sum_{t=1}^{T} e_{i t}^{2}-\frac{1}{n T} \sum_{i=1}^{n} \sum_{t=1}^{T} e_{i t}^{2}\right)^{2}
$$

approximately for large $T$ and $n$, which is the sample analog of $\frac{1}{8}\left(\sigma_{\lambda}^{2}+\sigma_{\varepsilon}^{2}\right)^{-3 / 2} \operatorname{var}\left(\sigma_{\varepsilon i}^{2}\right)$ mentioned in Remark 3. Based on equation (12) we propose a simple test for the equality of $R M S E_{R T}$ and $R M S E_{L P S}$ for large $T$ and $n$ as in the following limit theorem.

Theorem 3. Suppose Assumptions 1-4 hold. Then under the null hypothesis that 


$$
\begin{aligned}
\frac{1}{n} \sum_{i=1}^{n}\left(\sigma_{\varepsilon i}^{2}-\frac{1}{n} \sum_{i=1}^{n} \sigma_{\varepsilon i}^{2}\right)^{2} & =o\left(T^{-1} n^{-1}\right), \\
W & =\frac{Y_{n T}^{\hat{\beta}}-\widehat{Y}_{n T}^{\hat{\beta}}-\frac{1}{2} \hat{\beta}(\hat{\beta}-1) \widehat{Y}_{n T}^{\hat{\beta}-2}}{\hat{\beta} \widehat{Y}_{n T}^{\hat{\beta}-1}} \rightarrow_{d} N(0,1),
\end{aligned}
$$

as $(n, T \rightarrow \infty)$ and $\frac{n}{T} \rightarrow 0$, where $Y_{n T}=\frac{1}{s_{n T}} \sum_{i=1}^{n}\left(T\left(\frac{1}{T} \sum_{t=1}^{T} e_{i t}^{2}-\frac{1}{n T} \sum_{i=1}^{n} \sum_{t=1}^{T} e_{i t}^{2}\right)^{2}\right), \widehat{Y}_{n T}=$ $\frac{1}{s_{n T}} \sum_{i=1}^{n}\left(\frac{n-1}{n}\right)^{2} \widetilde{\Omega}_{i T}$, and $\hat{\beta}=1-\frac{2}{3} \frac{\left(\sum_{i=1}^{n} \widetilde{\Omega}_{i T}\right)\left(\sum_{i=1}^{n} \widetilde{\Omega}_{i T}^{3}\right)}{\left(\sum_{i=1}^{n} \widetilde{\Omega}_{i T}^{2}\right)^{2}}$ with $s_{n T}^{2}=2 \sum_{i=1}^{n}\left(\frac{n-1}{n}\right)^{2} \widetilde{\Omega}_{i T}, \widetilde{\Omega}_{i T}=\widetilde{\omega}_{\varepsilon i T}+$ $4 \widetilde{\sigma}_{\lambda}^{2} \widetilde{\sigma}_{\varepsilon i}^{2}, \tilde{\sigma}_{\varepsilon i}^{2}=\left(\frac{n}{n-1}\right)^{2} \widehat{\sigma}_{\varepsilon i}^{2}-\left(\frac{n}{n-1}\right)^{2} \frac{1}{n^{2}} \sum_{j \neq i}^{n} \widehat{\sigma}_{\varepsilon j}^{2}, \widetilde{\sigma}_{\lambda}^{2}=\widehat{\sigma}_{\lambda}^{2}-\frac{1}{n^{2}} \sum_{i=1}^{n} \widehat{\sigma}_{\varepsilon i}^{2}$.

Remark 6. Theorem 3 establishes the joint limit distribution of the statistic $W$ for testing the null hypothesis that $\frac{1}{n} \sum_{i=1}^{n}\left(\sigma_{\varepsilon i}^{2}-\frac{1}{n} \sum_{i=1}^{n} \sigma_{\varepsilon i}^{2}\right)^{2}=o\left(T^{-1} n^{-1}\right)$ rather than $\operatorname{var}\left(\sigma_{\varepsilon i}^{2}\right)=0$. The proof of Theorem 3 is obtained by first establishing the asymptotic distribution for the case $\hat{\beta}=1$ and then by applying Taylor's expansion. Note that one could use the statistic for the case $\hat{\beta}=1$ to test the null hypothesis. ${ }^{6}$ Such a test statistic, however, suffers from both severe size distortions because $\left[T\left(\frac{1}{T} \sum_{t=1}^{T} e_{i t}^{2}-\frac{1}{n T} \sum_{i=1}^{n} \sum_{t=1}^{T} e_{i t}^{2}\right)^{2}\right]$ follows an asymptotic chi-square distribution as $T \rightarrow \infty$ and slow convergence to standard normality due to its highly right skewness (see, for example, Chen and Deo (2004)). Following Chen and Deo (2004)'s suggestion, we apply general power transformation to address these issues and obtain the $W$ statistic, which encompasses the Wilson-Hilferty cube root transformation as a special case where $\hat{\beta}=1 / 3$ (a result implied by the equality of $\widetilde{\Omega}_{i T}$ across $i$ ). Our test for forecaster homogeneity differs from the Lagrange multiplier test for heteroskedasticity developed by Baltagi, et al. (2006) in that we do not assume that the idiosyncratic variance is a function of some known covariates.

Remark 7. The scaling factor $\left(\frac{n-1}{n}\right)^{2}$ and the employment of both $\widetilde{\sigma}_{\varepsilon i}^{2}$ and $\tilde{\sigma}_{\lambda}^{2}$ (when com-

\footnotetext{
${ }^{6} \mathrm{~A}$ similar approach was adopted by Pesaran and Yamagata (2008) in their test of slope homogeneity in large random coefficient panel data models.
} 
pared to $\widehat{\sigma}_{\varepsilon i}^{2}$ and $\widehat{\sigma}_{\lambda}^{2}$ ) are used to reduce the asymptotic bias of their respective estimators in panel estimation. The restriction $\frac{n}{T} \rightarrow 0$ controls for the effect of approximation errors and prevents the asymptotic bias from having a non-vanishing impact on the test statistic $W$.

Remark 8. We need to point out that Theorems 2 and 3 test two different layers of heterogeneity with regard to the idiosyncratic error $\varepsilon_{i t}$. Under the null hypothesis in Theorem 2, the idiosyncratic shocks do not contribute to the measure of forecast uncertainty - they are insignificant compared to the variance of the common shock. In contrast, under the null hypothesis in Theorem 3, the variance of the idiosyncratic error is a significant part of forecast uncertainty. Essentially, Theorem 3 tests whether the variances of idiosyncratic errors are identical and this second layer of heterogeneity is a necessary and sufficient condition for the difference between $R M S E_{R T}$ and $R M S E_{L P S}$.

Although in theory the appropriate power transformation helps to minimize the size distortion, our simulation study indicates that the test based on Theorem 3 is slightly oversized (see Table 1). These results are consistent with the simulation findings in Chen and Deo (2004) that attribute the modest oversize problem to parameter estimation uncertainty. Table 2 shows that the transformed test $W$ has satisfactory power provided that the average of idiosyncratic variances is not too small relative to the variance of common shocks. The test becomes more powerful when the proportion of idiosyncratic variances that differs from the average of idiosyncratic variances increases. However, the power tends to fall as $n$ rises for given $T$. This result seems counterintuitive since the power generally increases with the size of the cross section dimension in panel models. But a careful examination of the test statistic reveals that there is a negative bias term of order $O_{p}\left(\frac{n}{T}\right)$ in the process of approximation of the test statistic, contributing to the decreasing power for given $T$. The impact of this negative bias term becomes negligible provided that $T$ grows faster than $n$ as confirmed in Table 2 . 
It is interesting to note that, when idiosyncratic error variances are the same across forecasters, both $R M S E_{R T}$ and $R M S E_{L P S}$ can be interpreted as measuring the uncertainty of a typical forecast in the panel. This interpretation is consistent with the explicitly stated objective in Reifschneider and Tulip (2007) that the benchmark uncertainty figure they are providing is that of a typical forecaster in the pool that will be individualized by FOMC members based on their own special experiences. To illustrate, let us assume that in predicting $A_{t}$, each forecaster possesses two types of information: (i) common information, $y_{t}=A_{t}+\lambda_{t}$ with precision $\kappa_{t}$; and (ii) private information, $z_{i t}=A_{t}+\varepsilon_{i t}$ with precision $\tau_{t}$, where $\lambda_{t}$ and $\varepsilon_{i t}$ are mutually independent and normally distributed with mean 0 . Then according to Bayes rule, individual $i$ 's forecast $F_{i t}$ is a weighted average of the two signals with their precision as the weights

$$
F_{i t} \equiv E\left[A_{t} \mid y_{t}, z_{i t}\right]=\frac{\kappa_{t} y_{t}+\tau_{t} z_{i t}}{\kappa_{t}+\tau_{t}}
$$

and its associated uncertainty is simply $\frac{1}{\kappa_{t}+\tau_{t}}$. Taking expectations on both sides conditional on available information, the left-hand side of equation (3) becomes

$$
\frac{1}{n} \sum_{i=1}^{n} E\left[\left(A_{t}-F_{i t}\right)^{2} \mid y_{t}, z_{i t}\right]=\frac{1}{n} \sum_{i=1}^{n} E\left[\left(\frac{\kappa_{t} \lambda_{t}+\tau_{t} \varepsilon_{i t}}{\kappa_{t}+\tau_{t}}\right)^{2} \mid y_{t h}, z_{i t}\right]=\frac{1}{\kappa_{t}+\tau_{t}}
$$

Thus, we see that our uncertainty measure, $R M S E_{L P S}$, is the same as the variance of an individual forecast, with both of them equal to $\frac{1}{\kappa_{t}+\tau_{t}}$ in the population. So it can be interpreted as the confidence an outside observer will have in a randomly drawn typical individual forecast from the panel, as pointed out by Giordani and Söderlind (2003). Also note that the expected value of the first term on the right hand side of equation (3) can be expressed as

$$
E\left[\left(A_{t}-F_{\cdot t}\right)^{2} \mid y_{t}, z_{i t}\right]=\frac{\kappa_{t}+\frac{\tau_{t}}{n}}{\left(\kappa_{t}+\tau_{t}\right)^{2}}
$$


For $n>1$, it is easy to verify that $\frac{\kappa_{t}+\frac{\tau_{t}}{n}}{\left(\kappa_{t}+\tau_{t}\right)^{2}}$ in equation (15) is less than $\frac{1}{\kappa_{t}+\tau_{t}}$ in equation (14). This shows that the uncertainty conventionally associated with the consensus forecast, $R M S E_{A F}$, is less than $R M S E_{L P S}$ or $R M S E_{R T}$. However, as we have shown before, when idiosyncratic errors are heteroskedastic, $R M S E_{R T}$ will be in general smaller than $R M S E_{L P S}$ implying that $R M S E_{R T}$ does not fully incorporate disagreement among the forecasters.

\section{Illustration of Underestimation of Uncertainty}

In this section, we present estimates of historical uncertainty in inflation and output growth forecasts using $R M S E_{L P S}$, and compare it to $R M S E_{A F}$ and $R M S E_{R T}$. The data in our study are taken from the Survey of Professional Forecasters and Real Time Data Set for Macroeconomists (RTDSM), provided by the Federal Reserve Bank of Philadelphia. The survey asks for the forecasts of inflation and output growth for both the current year and next year, giving us information on eight quarterly forecasts. In our analysis, inflation is measured as the annual-average over annual-average percent change of GDP deflator, and output growth is similarly defined in terms of real GDP. The actual horizons for these forecasts are approximately $7 \frac{1}{2}, 6 \frac{1}{2}, \ldots, \frac{1}{2}$ quarters but we refer to them simply as quarterly horizons of $8,7, \ldots, 1$.

In calculating the percentage change from year $t-1$ to $t$, we transform the quarterly forecasts of the level of the variables into annual forecasts of the growth rate of the variables. Specifically, we use the forecasts of the level of the variables in the current and subsequent quarters and the actual values from the vintage of data available at that time to calculate the forecast of annual inflation and output growth for the current year. For example, in the second quarter of the current year, a respondent would use data on the actual values of real GDP in the first quarter $\left(Y_{1, t}\right)$ and make predictions through the end of year $t\left(F_{2, t}, F_{3, t}, F_{4, t}\right)$. Accordingly, as an example, the output growth forecast for the current year at horizon of 3 
quarters is calculated as $f_{t}=\left(Y_{1, t}+F_{2, t}+F_{3, t}+F_{4, t}\right) /\left(Y_{1, t-1}+Y_{2, t-1}+Y_{3, t-1}+Y_{4, t-1}\right)$, where $Y_{1, t-1}$ through $Y_{4, t-1}$ are the level of actual real GDP in the first through fourth quarter of the year $t-1$, which are available to forecasters in the second quarter of year $t$. To calculate the percentage change from year $t$ to $t+1$, we simply divide $F_{t+1}$ by $F_{t}$, where $F_{t+1}$ and $F_{t}$ are the annual forecasts of real GDP for the next year and current year, respectively. To calculate forecast errors, we choose the first quarterly release of annual inflation and output growth to compute the actual values.

A practical challenge that arises in using SPF dataset is the substantial gaps in the panel of forecasts, reflecting non-responses by existing participants, and the frequent entry and exit of some participants. To reduce sampling distortions associated with missing values in the raw dataset, we focus on the forecasts made from 1991:Q1 until 2013:Q4 following Engelberg, et al. (2011) and filter the data to include only those forecasters who have responded at least $40 \%$ of time. Even after filtering out irregular respondents, the data have some missing values. To impute these missing values, we follow Genre, et al. (2013) to run the following panel regression:

$$
F_{i t}-F_{\cdot t}=\beta_{i}\left(F_{i, t-1}-F_{\cdot t-1}\right)+\epsilon_{i t},
$$

where $F_{. t}=\frac{1}{n} \sum_{j=1}^{n} F_{i t}$. The missing values for forecaster $i$ in period $t$ are replaced with the period $t$ average forecast plus a fraction of the previously observed deviation from the average for $\beta_{i}<1$ or with the previously reported individual forecast plus the change in the average for $\beta_{i}=1$.

Tables 3 and 4 report the results for inflation and output growth forecasts, respectively, using the balanced panels. Three points are worth noting. First, the RMSEs associated with output are uniformly higher compared to inflation due to a differential incidence of common shocks. This phenomenon, which makes real GDP growth a difficult variable to predict, 
has been documented by Lahiri and Sheng (2008) using a heterogeneous learning model. Second, among the three measures $R M S E_{A F}$ yields the smallest forecast uncertainty and there is a sizable difference between $R M S E_{A F}$ and the other two. For all eight horizons, the idiosyncratic errors are important, as shown by significant test statistics $Z$. Thus, pervasive heterogeneity seems to exist amongst professional forecasters but is ignored in the traditional measure $R M S E_{A F}$. Simple calculations show that when $R M S E_{A F}$ is used as the measure of historical uncertainty, the degree of underestimation is between $8 \%$ and $19 \%$ for inflation forecasts and between $1 \%$ and $8 \%$ for output growth forecasts.

Third, for all eight horizons $R M S E_{R T}$ is less than $R M S E_{L P S}$. Yet, the differences between these two measures are relatively small and often not statistically different from zero. Note that the RMSE figures that are reported by Reifschneider and Tulip (2007) and those in this paper are not directly comparable. We present average year-over-year forecasts whereas RT use Q4 over Q4 percent changes. The latter target tends to be more variable. More importantly, RT used the simple averages of the individual projections in SPF, Blue Chip and FOMC panels, together with Greenbook, Congressional Budget Office (CBO) and the Administration forecasts giving $n=6$ in their calculations. More generally, their measure is expressed as $R M S E^{\text {group }}=\frac{1}{M} \sum_{m=1}^{M} \sqrt{\frac{1}{T} \sum_{t=1}^{T}\left(A_{t}-F_{\cdot t}^{m}\right)^{2}}$, where $F_{\cdot t}^{m}$ is the mean forecast for the group $m$, for the target year $t$ and $h$-period ahead to the end of the target year. By averaging across individual projections, most of idiosyncratic differences and disagreement in FOMC, SPF and Blue Chip forecasts have inadvertently been washed away. They found very little heterogeneity in these six forecasts. On the other hand, their simultaneous use of Greenbook, CBO, Adminstration, mean FOMC, SPF, and Blue Chip forecasts meant that RT had to meticulously sort out important differences in the comparability of these six forecasts due to data coverage, timing of forecasts, reporting basis for projections, and forecast conditionality. Despite all these differences, these two sets of uncertainty estimates are very close in the context of SPF dataset. At least a part of the explanation for this similarity is 
due to the use of dataset from professional forecasters. For non-professional forecasters, such as surveys of households, where the idiosyncratic errors are heteroskedastic, we expect a substantial difference between RT and LPS uncertainty measures. Indeed, if the cross sectional

variance of idiosyncratic error variances, defined as $\frac{1}{n} \sum_{i=1}^{n}\left(\frac{1}{T} \sum_{t=1}^{T} e_{i t}^{2}-\frac{1}{n T} \sum_{i=1}^{n} \sum_{t=1}^{T} e_{i t}^{2}\right)^{2}$, were to increase from 0.0004 to 0.004 at 1-quarter ahead inflation forecast, $R M S E_{R T}$ would decrease from 0.209 in Table 3 to 0.163 , resulting in an underestimation of the correct benchmark uncertainty by $23 \%$.

Despite our effort in imputing the missing values in the survey data to maintain the underlying cross section variability, the imputed data sets will invariably reduce the heterogeneity of forecasts, as pointed out by Lahiri, et al. (2015). As an alternative experiment to avoid the missing data problem, we construct a pseudo balanced panel of 21 forecasters with each of them representing the minimum, 5th percentile, 10th percentile, ..., 95th percentile and the maximum of individual forecasts, respectively. The results, reported in Tables 5 and 6, clearly show that, as theoretically expected, $R M S E_{A F}<R M S E_{R T}<R M S E_{L P S}$ at all eight horizons in both inflation and output growth forecasts. The differences between $R M S E_{A F}$ and $R M S E_{L P S}$ for all horizons and both variables are statistically significant. When $R M S E_{A F}$ is used as the measure of historical uncertainty the degree of underestimation is between $4 \%$ and $23 \%$ in inflation forecasts and between $2 \%$ and $12 \%$ in output growth forecasts. As before, the differences between $R M S E_{R T}$ and $R M S E_{L P S}$ are relatively small. But some of these differences are now statistically significant, particularly at longer horizons.

\section{Concluding Remarks}

A number of surveys of professional forecasters and households are regularly conducted in many countries around the world, and a widespread interest in these surveys suggests that 
the aggregate macroeconomic forecasts reported by these organizations are considered useful by policy makers, investors and other stakeholders. Even though it is now recognized in the forecasting profession that an average forecast by itself is of limited use and should be reported with an indication of the associated uncertainty, currently the consensus forecasts from these surveys are not reported with uncertainty bands.

The dominant methodology of forecast combination in econometrics is due to Bates and Granger (1969) whose basic criterion for optimal combination is based on minimizing the mean square error of combined forecasts that rule out any consideration of the cross sectional distribution of forecasts. Thus, an increase in forecaster discord, ceteris paribus, will have no effect on the uncertainty of the combined forecast. By reformulating the loss function as minimization of the risk averaged over all possible forecasts rather than minimizing the risk of the average forecast, disagreement among forecasters can be made a component of the aggregate uncertainty. Using a standard factor decomposition of forecast errors, we have shown that the aggregate forecast uncertainty is composed of disagreement among forecasters plus the volatility of the common shock. This result is consistent with the recent model averaging literature that the risk associated with a combined forecast should incorporate not only the average of the variances of individual forecasts but also the variance of the point forecasts across different models. Even if we have highly precise individual forecasts, we might end up with considerable uncertainty about the combined forecast if these point forecasts are very different across forecasters. The key is to realize that individual risks should be integrated into the combined forecast and, as a result, the risk faced by a policy maker in using the average forecast is the risk associated with the use of the forecast of a typical member of the panel, rather than the variance of the average forecast.

Using two new statistics to test the homogeneity of idiosyncratic errors at two layers, we find significant heterogeneity in professional forecasters, even though the variances of the idiosyncratic errors are not significantly different. The first layer of heterogeneity suggests 
that the uncertainty measure implied by the Bates-Granger approach substantially underestimates the true forecast uncertainty of the average forecast. However, due to the observed lack of heterogeneity in the individual error variances, the benchmark uncertainty formula suggested by Reifschneider and Tulip (2007) does not seem to induce significant underestimation of uncertainty. In retrospect, lack of the second layer of heterogeneity is not entirely unexpected given that the SPF members are all experienced experts who are forecasting two closely monitored macro variables using widely available public information.

Citing frequent entry and exit of survey forecasters who tend to be highly heterogeneous, Engelberg, et al. (2011) have advised against the use of consensus forecasts altogether, even though they indicated that this practice will possibly not be discontinued in the near future. Consistent with this sentiment, our suggested measure of uncertainty of the consensus forecast recognizes this well-established heterogeneity among forecasters of the panel together with its changing composition, and will help interpreting movements in the consensus forecasts with appropriate confidence bands. Often the consensus forecasts in surveys like the Blue Chip are reported together with maximum, minimum and interquartile range of the point forecasts, presumably to suggest the underlying uncertainty. However, as we have explained, these features of the cross sectional distribution indicate only a part of the aggregate forecast uncertainty.

One potential concern in incorporating disagreement as part of aggregate uncertainty is that the prediction intervals will get wider, making intertemporal movements in consensus forecasts less meaningful. Why would practitioners opt for enlarged confidence bands when they are less likely to obtain news-worthy results? The simple answer is that in the long run the reported forecasts will be more credible and the uncertainty measures better calibrated. As aptly put by Draper (1995) in his concluding remark, "which is worse - widening the bands now or missing the truth later?" 


\section{Appendix}

We start with some useful lemmas.

Lemma 1. Suppose Assumptions 1-4 hold, then as $(n, T \rightarrow \infty)$

(a) $\frac{1}{\sqrt{T}} \sum_{t=1}^{T}\left(\lambda_{t}^{2}-\sigma_{\lambda}^{2}\right) \rightarrow_{d} N\left(0, \Sigma_{\lambda}\right)$.

(b) $\frac{1}{T^{1 / 2}} \sum_{t=1}^{T} \mu_{i t} \lambda_{t}=O_{p}\left(T^{-1 / 4} n^{-\alpha / 4}\right) ; \frac{1}{T^{1 / 2}} \sum_{t=1}^{T} \mu_{i t} \varepsilon_{j t}=O_{p}\left(T^{-1 / 4} n^{-\alpha / 4}\right)$.

(c) $\frac{1}{\sqrt{T}} \sum_{t=1}^{T} \lambda_{t} \varepsilon_{i t} \rightarrow_{d} N\left(0, \sigma_{\lambda}^{2} \sigma_{\varepsilon i}^{2}\right) ; \frac{1}{\sqrt{n T}} \sum_{i=1}^{n} \sum_{t=1}^{T} \lambda_{t} \varepsilon_{i t} \rightarrow_{d} N\left(0, \sigma_{\lambda}^{2} \sigma_{\varepsilon}^{2}\right)$ with $\sigma_{\varepsilon}^{2}=\lim _{n \rightarrow \infty} \frac{1}{n} \sum_{i=1}^{n} \sigma_{\varepsilon i}^{2}$.

(d) $\frac{1}{\sqrt{T}} \sum_{t=1}^{T}\left(\varepsilon_{i t}^{2}-\sigma_{\varepsilon i}^{2}\right) \rightarrow_{d} N\left(0, \omega_{\varepsilon i}\right) ; \frac{1}{\sqrt{n T}} \sum_{t=1}^{T} \sum_{i=1}^{n}\left(\varepsilon_{i t}^{2}-\sigma_{\varepsilon i}^{2}\right) \rightarrow_{d} N\left(0, \omega_{\varepsilon}\right)$ with $\omega_{\varepsilon}=\lim _{n \rightarrow \infty} \frac{1}{n} \sum_{i=1}^{n} \omega_{\varepsilon i}$.

(e) $\frac{1}{\sqrt{T}} \sum_{t=1}^{T}\left[\left(\frac{1}{\sqrt{n}} \sum_{j \neq i}^{n} \varepsilon_{j t}\right)^{2}-\frac{1}{n} \sum_{j \neq i}^{n} \sigma_{\varepsilon j}^{2}\right] \rightarrow_{d} N\left(0,2 \sigma_{\varepsilon}^{4}\right), \frac{1}{\sqrt{n T}} \sum_{t=1}^{T} \varepsilon_{i t} \sum_{j \neq i}^{n} \varepsilon_{j t} \rightarrow_{d} N\left(0, \Sigma_{\varepsilon}\right)$ where $\Sigma_{\varepsilon}=\sigma_{\varepsilon i}^{2} \sigma_{\varepsilon}^{2}$.

(f) $\frac{1}{\sqrt{T}} \sum_{t=1}^{T}\left[\left(\varepsilon_{i t}^{2}-\sigma_{\varepsilon i}^{2}\right)+2 \lambda_{t} \varepsilon_{i t}\right] \rightarrow_{d} N\left(0, \omega_{\varepsilon i}+4 \sigma_{\lambda}^{2} \sigma_{\varepsilon i}^{2}\right) ; \frac{1}{\sqrt{n T}} \sum_{i=1}^{n} \sum_{t=1}^{T}\left[\left(\varepsilon_{i t}^{2}-\sigma_{\varepsilon i}^{2}\right)+2 \lambda_{t} \varepsilon_{i t}\right] \rightarrow_{d}$ $N\left(0, \omega_{\varepsilon}+4 \sigma_{\lambda}^{2} \sigma_{\varepsilon}^{2}\right)$.

Proof. (a) By Assumption 2 and the independence of white noise shocks $\zeta_{t k}$ of $\lambda_{t}, \lambda_{t}^{2}-\sigma_{\lambda}^{2}$ is a stationary ergodic (centered) process with $E\left\{\left(\lambda_{t}^{2}-\sigma_{\lambda}^{2}\right)\left(\lambda_{t-k}^{2}-\sigma_{\lambda}^{2}\right)\right\}=0$ for all $k>h-1$. Also by Loeve's $c_{r}$ inequality, we have $E\left|\lambda_{t}^{2}-\sigma_{\lambda}^{2}\right|^{2+\frac{\delta}{2}} \leq 2^{1+\frac{\delta}{2}}\left(E\left|\lambda_{t}\right|^{4+\delta}+\sigma_{\lambda}^{4+\delta}\right)<\infty$ for some $\delta>0$.Therefore $\frac{1}{\sqrt{T}} \sum_{t=1}^{T}\left(\lambda_{t}^{2}-\sigma_{\lambda}^{2}\right) \rightarrow_{d} N\left(0, \Sigma_{\lambda}\right)$ by virtue of Theorem 5.6 in Hall and Heyde (1980) since $\operatorname{var}\left(\frac{1}{\sqrt{T}} \sum_{t=1}^{T} \lambda_{t}^{2}\right) \rightarrow \Sigma_{\lambda}>0$ as $T \rightarrow \infty$.

(b) Let $\rho_{k}=\operatorname{corr}\left(\lambda_{t}, \lambda_{t+k}\right)$. Then by Assumptions 1 and 2, we have

$$
E\left(\frac{1}{T^{1 / 2}} \sum_{t=1}^{T} \mu_{i t} \lambda_{t}\right)=\frac{1}{T^{1 / 2}} \sum_{t=1}^{T} \mu_{i t} E\left(\lambda_{t}\right)=0
$$


and

$$
\begin{aligned}
E\left(\frac{1}{T^{1 / 2}} \sum_{t=1}^{T} \mu_{i t} \lambda_{t}\right)^{2} & =\sigma_{\lambda}^{2} \frac{1}{T} \sum_{t=1}^{T} \mu_{i t}^{2}+\sigma_{\lambda}^{2} \frac{1}{T} \sum_{t=1}^{T-k} \sum_{k=1}^{h-1} \rho_{k} \mu_{i t} \mu_{i(t+k)} \\
& \leq \sigma_{\lambda}^{2} \frac{1}{T} \sum_{t=1}^{T} \mu_{i t}^{2}+\sigma_{\lambda}^{2} \sum_{k=1}^{h-1}\left(\frac{1}{T} \sum_{t=1}^{T} \mu_{i t}^{2}\right)^{1 / 2}\left(\frac{1}{T} \sum_{t=1}^{T-k} \mu_{i(t+k)}^{2}\right)^{1 / 2} \\
& \leq h \sigma_{\lambda}^{2}\left(\frac{1}{T} \sum_{t=1}^{T} \mu_{i t}^{4}\right)^{1 / 2} \\
& =O\left(T^{-1 / 2} n^{-\alpha / 2}\right)
\end{aligned}
$$

where the first inequality is obtained by virtue of Cauchy Schwarz inequality and the fact that $\rho_{k} \leq 1$ for all $k$ while the second inequality follows from Jensen inequality. It follows immediately that $\frac{1}{T^{1 / 2}} \sum_{t=1}^{T} \mu_{i t} \lambda_{t}=O_{p}\left(T^{-1 / 4} n^{-\alpha / 4}\right)$. Similarly, we can show that $\frac{1}{T^{1 / 2}} \sum_{t=1}^{T} \mu_{i t} \varepsilon_{j t}=O_{p}\left(T^{-1 / 4} n^{-\alpha / 4}\right)$.

(c) By Assumptions 2, 3 and 4, $\lambda_{t} \varepsilon_{i t}$ is stationary and ergodic for each $i$ with $E\left(\lambda_{t} \varepsilon_{i t}\right)=0$, $E\left(\lambda_{t}^{2} \varepsilon_{i t}^{2}\right)=\sigma_{\lambda}^{2} \sigma_{\varepsilon i}^{2}$ and $E\left(\lambda_{t} \varepsilon_{i t} \lambda_{t-k} \varepsilon_{i(t-k)}\right)=0$ for all $k \geq 1$. In addition, $E\left|\lambda_{t} \varepsilon_{i t}\right|^{2+\delta}<\infty$ by Liapunov's inequality and the independence of $\lambda_{t}$ and $\varepsilon_{i t}$. Hence it follows from Theorem 5.6 in Hall and Heyde (1980) that for each $i$

$$
\frac{1}{\sqrt{T}} \sum_{t=1}^{T} \lambda_{t} \varepsilon_{i t} \rightarrow_{d} N\left(0, \sigma_{\lambda}^{2} \sigma_{\varepsilon i}^{2}\right),
$$

as $T \rightarrow \infty$. It remains to show

$$
\frac{1}{\sqrt{n T}} \sum_{i=1}^{n} \sum_{t=1}^{T} \lambda_{t} \varepsilon_{i t} \rightarrow_{d} N\left(0, \sigma_{\lambda}^{2} \sigma_{\varepsilon}^{2}\right),
$$


as $(n, T \rightarrow \infty)$. Let $\xi_{i n T}=\frac{1}{\sqrt{T} \sigma_{\lambda}\left(\sum_{i=1}^{n} \sigma_{\varepsilon i}^{2}\right)^{1 / 2}} \sum_{t=1}^{T} \lambda_{t} \varepsilon_{i t}$. Then

$$
\begin{aligned}
& \sum_{i=1}^{n} E\left[\xi_{i n T}^{2} 1\left\{\left|\xi_{i n T}\right|>\epsilon\right\}\right] \\
\leq & \sigma_{\lambda}^{-2}\left(\inf _{i} \sigma_{\varepsilon i}^{2}\right)^{-1} \sup _{i, T} E\left[\left(\frac{1}{\sqrt{T}} \sum_{t=1}^{T} \lambda_{t} \varepsilon_{i t}\right)^{2} 1\left\{\frac{\left|\frac{1}{\sqrt{T}} \sum_{t=1}^{T} \lambda_{t} \varepsilon_{i t}\right|}{\sigma_{\lambda}\left(\inf _{i} \sigma_{\varepsilon i}^{2}\right)^{1 / 2}}>n^{1 / 2} \epsilon\right\}\right] \\
\rightarrow & 0
\end{aligned}
$$

as $n \rightarrow \infty$ because $\left(\frac{1}{\sqrt{T}} \sum_{t=1}^{T} \lambda_{t} \varepsilon_{i t}\right)^{2}$ is uniformly integrable in $T$, as implied by Assumptions 2 and 3. So $\sum_{i=1}^{n} \xi_{i n T} \rightarrow_{d} N(0,1)$ as $(n, T \rightarrow \infty)$ in view of Theorem 2 in Phillips and Moon (1999), from which we can conclude that $\frac{1}{\sqrt{n T}} \sum_{i=1}^{n} \sum_{t=1}^{T} \lambda_{t} \varepsilon_{i t} \rightarrow_{d} N\left(0, \sigma_{\lambda}^{2} \sigma_{\varepsilon}^{2}\right)$ as $(n, T \rightarrow \infty)$.

(d) and (e). The proofs of (d) and (e) are similar to that of (c) and thus omitted here.

(f). The proof of (f) follows directly from (c), (d) and the independence of $\lambda_{t} \varepsilon_{i t}$ and $\left(\varepsilon_{i t}^{2}-\sigma_{\varepsilon i}^{2}\right)$ over $t$ and across $i$.

Lemma 2. Suppose Assumptions 1-4 hold, then as $(n, T \rightarrow \infty)$

(a) $\frac{1}{\sqrt{T}} \sum_{t=1}^{T}\left(\mu_{i t}-\frac{1}{n} \sum_{i=1}^{n} \mu_{i t}\right)^{2}=O\left(n^{-\alpha / 2}\right) ; \frac{1}{\sqrt{T}} \sum_{t=1}^{T}\left(\mu_{i t}-\frac{1}{n} \sum_{i=1}^{n} \mu_{i t}\right)^{4}=O\left(T^{-1 / 2} n^{-\alpha}\right)$.

(b) $\frac{1}{\sqrt{T}} \sum_{t=1}^{T}\left(\mu_{i t}-\frac{1}{n} \sum_{i=1}^{n} \mu_{i t}\right)^{2}\left(\varepsilon_{i t}-\frac{1}{n} \sum_{i=1}^{n} \varepsilon_{i t}\right)^{2}=O_{p}\left(n^{-\alpha / 2}\right)$.

(c) $\frac{1}{\sqrt{T}} \sum_{t=1}^{T}\left(\mu_{i t}-\frac{1}{n} \sum_{i=1}^{n} \mu_{i t}\right)^{3}\left(\varepsilon_{i t}-\frac{1}{n} \sum_{i=1}^{n} \varepsilon_{i t}\right)=O_{p}\left(T^{-1 / 4} n^{-3 \alpha / 4}\right)$.

(d) $\frac{1}{\sqrt{T}} \sum_{t=1}^{T}\left(\mu_{i t}-\frac{1}{n} \sum_{i=1}^{n} \mu_{i t}\right)\left(\varepsilon_{i t}-\frac{1}{n} \sum_{i=1}^{n} \varepsilon_{i t}\right)=O_{p}\left(T^{-1 / 4} n^{-\alpha / 4}\right)$.

(e) $\frac{1}{\sqrt{T}} \sum_{t=1}^{T}\left(\mu_{i t}-\frac{1}{n} \sum_{i=1}^{n} \mu_{i t}\right)\left(\varepsilon_{i t}-\frac{1}{n} \sum_{i=1}^{n} \varepsilon_{i t}\right)^{3}=O\left(T^{-1 / 4} n^{-\alpha / 4}\right)$.

(f) $\frac{1}{\sqrt{T}} \sum_{t=1}^{T}\left(\varepsilon_{i t}^{2}-\sigma_{\varepsilon i}^{2}\right)^{2}\left[\left(\frac{1}{\sqrt{n}} \sum_{j \neq i}^{n} \varepsilon_{j t}\right)^{2}-\frac{1}{n} \sum_{j \neq i}^{n} \sigma_{\varepsilon i}^{2}\right]=O_{p}(1)$. 
(g) $\frac{1}{\sqrt{T}} \sum_{t=1}^{T}\left\{\left(\varepsilon_{i t}^{2}-\sigma_{\varepsilon i}^{2}\right)^{2} \varepsilon_{i t}\left(\frac{1}{\sqrt{n}} \sum_{j \neq i}^{n} \varepsilon_{j t}\right)\right\}=O_{p}(1)$.

(h) $\frac{1}{\sqrt{T}} \sum_{t=1}^{T}\left[\left(\frac{1}{\sqrt{n}} \sum_{j \neq i}^{n} \varepsilon_{j t}\right)^{2}-\frac{1}{n} \sum_{j \neq i}^{n} \sigma_{\varepsilon i}^{2}\right]\left(\frac{1}{\sqrt{n}} \sum_{j \neq i}^{n} \varepsilon_{j t}\right) \varepsilon_{i t}=O_{p}(1)$.

(i) $\frac{1}{\sqrt{T}} \sum_{t=1}^{T}\left\{\left[\left(\frac{1}{\sqrt{n}} \sum_{j \neq i}^{n} \varepsilon_{j t}\right)^{2}-\frac{1}{n} \sum_{j \neq i}^{n} \sigma_{\varepsilon i}^{2}\right]^{2}-\xi_{n}\right\}=O_{p}(1)$ with $\xi_{n}=E\left[\left(\frac{1}{\sqrt{n}} \sum_{j \neq i}^{n} \varepsilon_{j t}\right)^{2}-\frac{1}{n} \sum_{j \neq i}^{n} \sigma_{\varepsilon i}^{2}\right]^{2}$.

(j) $\frac{1}{\sqrt{T}} \sum_{t=1}^{T}\left\{\varepsilon_{i t}^{2}\left(\frac{1}{\sqrt{n}} \sum_{j \neq i}^{n} \varepsilon_{j t}\right)^{2}-\sigma_{\varepsilon i}^{2}\left(\frac{1}{n} \sum_{j \neq i}^{n} \sigma_{\varepsilon i}^{2}\right)\right\}=O_{p}(1)$.

Proof. (a) Using $2 a b \leq a^{2}+b^{2}$ and Jensen inequality, we have

$$
\begin{aligned}
\frac{1}{\sqrt{T}} \sum_{t=1}^{T}\left(\mu_{i t}-\frac{1}{n} \sum_{i=1}^{n} \mu_{i t}\right)^{2} & \leq 2\left(\sum_{t=1}^{T} \mu_{i t}^{4}\right)^{1 / 2}+2 \frac{1}{n} \sum_{i=1}^{n}\left(\sum_{t=1}^{T} \mu_{i t}^{4}\right)^{1 / 2} \\
& =O\left(n^{-\alpha / 2}\right) .
\end{aligned}
$$

Next, by Loeve's $c_{r}$ inequality and Jensen inequality,

$$
\begin{aligned}
\frac{1}{\sqrt{T}} \sum_{t=1}^{T}\left(\mu_{i t}-\frac{1}{n} \sum_{i=1}^{n} \mu_{i t}\right)^{4} & \leq 8 \sqrt{T}\left(\frac{1}{T} \sum_{t=1}^{T} \mu_{i t}^{4}+\frac{1}{n T} \sum_{i=1}^{n} \sum_{t=1}^{T} \mu_{i t}^{4}\right) \\
& =O\left(T^{-1 / 2} n^{-\alpha}\right) .
\end{aligned}
$$

(b) By Cauchy Schwarz inequality, we have

$$
\begin{aligned}
& \frac{1}{\sqrt{T}} \sum_{t=1}^{T}\left(\mu_{i t}-\frac{1}{n} \sum_{i=1}^{n} \mu_{i t}\right)^{2}\left(\varepsilon_{i t}-\frac{1}{n} \sum_{i=1}^{n} \varepsilon_{i t}\right)^{2} \\
\leq & \sqrt{T}\left[\frac{1}{T} \sum_{t=1}^{T}\left(\mu_{i t}-\frac{1}{n} \sum_{i=1}^{n} \mu_{i t}\right)^{4}\right]^{1 / 2}\left[\frac{1}{T} \sum_{t=1}^{T}\left(\varepsilon_{i t}-\frac{1}{n} \sum_{i=1}^{n} \varepsilon_{i t}\right)^{4}\right]^{1 / 2} \\
= & O_{p}\left(n^{-\alpha / 2}\right),
\end{aligned}
$$

in view of Lemma 2(a) and the result that $\frac{1}{T} \sum_{t=1}^{T}\left(\varepsilon_{i t}-\frac{1}{n} \sum_{i=1}^{n} \varepsilon_{i t}\right)^{4}=O_{p}(1)$ as implied by Assumption 3. 
(c) The result follows directly from Cauchy Schwarz inequality and Lemma 2(a) and 2(b).

(d) Using the similar argument as in Lemma $1(\mathrm{~b})$, we have $\frac{1}{\sqrt{T}} \sum_{t=1}^{T} \mu_{i t} \varepsilon_{j t}=O_{p}\left(T^{-1 / 4} n^{-\alpha / 4}\right)$, which, together with Lemma 1(b), yields the desired result.

(e) Again, following a similar argument as in Lemma 1(b) we can obtain that $\frac{1}{\sqrt{T}} \sum_{t=1}^{T} \mu_{i t} \varepsilon_{i t}^{3}=$ $O_{p}\left(T^{-1 / 4} n^{-\alpha / 4}\right)$. Then it follows that $\frac{1}{\sqrt{T}} \sum_{t=1}^{T} \mu_{i t}\left(\varepsilon_{i t}-\frac{1}{n} \sum_{i=1}^{n} \varepsilon_{i t}\right)^{3}=O_{p}\left(T^{-1 / 4} n^{-\alpha / 4}\right)$ because $\frac{1}{n} \sum_{i=1}^{n} \varepsilon_{i t}=O_{p}\left(n^{-1 / 2}\right)$ (implied by central limit theorem for iid random variable) is of smaller stochastic order than $\varepsilon_{i t}$. Therefore

$$
\begin{aligned}
& \frac{1}{\sqrt{T}} \sum_{t=1}^{T}\left(\varepsilon_{i t}-\frac{1}{n} \sum_{i=1}^{n} \varepsilon_{i t}\right)^{3}\left(\mu_{i t}-\frac{1}{n} \sum_{i=1}^{n} \mu_{i t}\right) \\
= & \frac{1}{\sqrt{T}} \sum_{t=1}^{T} \mu_{i t}\left(\varepsilon_{i t}-\frac{1}{n} \sum_{i=1}^{n} \varepsilon_{i t}\right)^{3}-\frac{1}{n \sqrt{T}} \sum_{j=1}^{n} \sum_{t=1}^{T} \mu_{j t}\left(\varepsilon_{i t}-\frac{1}{n} \sum_{i=1}^{n} \varepsilon_{i t}\right)^{3} \\
= & O\left(T^{-1 / 4} n^{-\alpha / 4}\right) .
\end{aligned}
$$

(f)-(j): The results in (f)-(j) can be established by proving their asymptotic normality respectively and such proofs are similar to that of Lemma 1(c) and thus omitted here.

Lemma 3. Suppose $\frac{1}{\sqrt{n}} \sum_{i=1}^{n}\left(\sigma_{\varepsilon i}^{2}-\sigma_{\varepsilon}^{2}\right)^{2}=o(1)$, then $\frac{1}{\sqrt{n}} \sum_{i=1}^{n}\left(\sigma_{\varepsilon i}^{2}-\frac{1}{n} \sum_{i=1}^{n} \sigma_{\varepsilon i}^{2}\right)^{2}=o(1)$.

Proof. Observe that

$$
\begin{aligned}
\frac{1}{\sqrt{n}} \sum_{i=1}^{n}\left(\sigma_{\varepsilon i}^{2}-\frac{1}{n} \sum_{i=1}^{n} \sigma_{\varepsilon i}^{2}\right)^{2} & =\frac{1}{\sqrt{n}} \sum_{i=1}^{n}\left(\left(\sigma_{\varepsilon i}^{2}-\sigma_{\varepsilon}^{2}\right)-\frac{1}{n} \sum_{i=1}^{n}\left(\sigma_{\varepsilon i}^{2}-\sigma_{\varepsilon}^{2}\right)\right)^{2} \\
& =\frac{1}{\sqrt{n}} \sum_{i=1}^{n}\left(\sigma_{\varepsilon i}^{2}-\sigma_{\varepsilon}^{2}\right)^{2}-\sqrt{n}\left(\frac{1}{n} \sum_{i=1}^{n}\left(\sigma_{\varepsilon i}^{2}-\sigma_{\varepsilon}^{2}\right)\right)^{2} .
\end{aligned}
$$

Then by triangle inequality and Jensen inequality, we have

$$
\left|\frac{1}{\sqrt{n}} \sum_{i=1}^{n}\left(\sigma_{\varepsilon i}^{2}-\frac{1}{n} \sum_{i=1}^{n} \sigma_{\varepsilon i}^{2}\right)^{2}\right| \leq 2\left|\frac{1}{\sqrt{n}} \sum_{i=1}^{n}\left(\sigma_{\varepsilon i}^{2}-\sigma_{\varepsilon}^{2}\right)^{2}\right|=o(1) .
$$

as $n \rightarrow \infty$. 
Lemma 4. Suppose Assumptions 1-4 hold with $\widehat{\sigma}_{\lambda}^{2}=\frac{1}{T} \sum_{t=1}^{T} \bar{e}_{\cdot t}^{2}, \widehat{\sigma}_{\varepsilon i}^{2}=\frac{1}{T} \sum_{t=1}^{T}\left(e_{i t}-\bar{e}_{\cdot t}\right)^{2}$ and $\widehat{\omega}_{\varepsilon i T}=\frac{1}{T} \sum_{t=1}^{T}\left(\left(e_{i t}-\bar{e}_{\cdot t}\right)^{2}-\frac{1}{T} \sum_{t=1}^{T}\left(e_{i t}-\bar{e}_{\cdot t}\right)^{2}\right)^{2}$, where $\bar{e}_{\cdot t}=\frac{1}{n} \sum_{i=1}^{n} e_{i t}$. Then as $(n, T \rightarrow \infty)$

(a) $\sqrt{T}\left(\widehat{\sigma}_{\lambda}^{2}-\sigma_{\lambda}^{2}-\frac{1}{n^{2}} \sum_{i=1}^{n} \sigma_{\varepsilon i}^{2}\right) \rightarrow_{d} N\left(0, \Sigma_{\lambda}\right)$.

(b) $\sqrt{T}\left(\widehat{\sigma}_{\varepsilon i}^{2}-\left(\frac{n-1}{n}\right)^{2} \sigma_{\varepsilon i}^{2}-\frac{1}{n^{2}} \sum_{j \neq i}^{n} \sigma_{\varepsilon j}^{2}\right) \rightarrow_{d} N\left(0, \omega_{\varepsilon i}\right)$.

(c) $\sqrt{T}\left(\widehat{\omega}_{\varepsilon i T}-\left(\frac{n-1}{n}\right)^{4} \omega_{\varepsilon i}-\frac{1}{n}\left(\frac{n-1}{n}\right)^{2} \sigma_{\varepsilon i}^{2}\left(\frac{1}{n} \sum_{j \neq i}^{n} \sigma_{\varepsilon j}^{2}\right)-\frac{1}{n^{2}} \xi_{n}\right) \rightarrow_{d} N\left(0, \operatorname{var}\left(\left(\varepsilon_{i t}^{2}-\sigma_{\varepsilon i}^{2}\right)^{2}\right)\right.$.

Proof. (a) Since $\widehat{\sigma}_{\lambda}^{2}=\frac{1}{T} \sum_{t=1}^{T} \bar{e}_{\cdot t}^{2}$, we have

$$
\begin{aligned}
\sqrt{T}\left(\widehat{\sigma}_{\lambda}^{2}-\sigma_{\lambda}^{2}-\frac{1}{n^{2}} \sum_{i=1}^{n} \sigma_{\varepsilon i}^{2}\right)= & \frac{1}{\sqrt{T}} \sum_{t=1}^{T}\left[\left(\frac{1}{n} \sum_{i=1}^{n} e_{i t}\right)^{2}-\sigma_{\lambda}^{2}-\frac{1}{n^{2}} \sum_{i=1}^{n} \sigma_{\varepsilon i}^{2}\right] \\
= & \frac{1}{\sqrt{T}} \sum_{t=1}^{T}\left(\frac{1}{n} \sum_{i=1}^{n} \mu_{i t}\right)^{2}+\frac{1}{\sqrt{T}} \sum_{t=1}^{T}\left(\lambda_{t}^{2}-\sigma_{\lambda}^{2}\right) \\
& +\frac{1}{n \sqrt{T}} \sum_{t=1}^{T}\left[\left(\frac{1}{\sqrt{n}} \sum_{i=1}^{n} \varepsilon_{i t}\right)^{2}-\frac{1}{n} \sum_{i=1}^{n} \sigma_{\varepsilon i}^{2}\right] \\
& +\frac{2}{n \sqrt{T}} \sum_{i=1}^{n} \sum_{t=1}^{T} \lambda_{t} \mu_{i t}+\frac{2}{n \sqrt{T}} \sum_{i=1}^{n} \sum_{t=1}^{T} \lambda_{t} \varepsilon_{i t} \\
& +\frac{2}{n^{2} \sqrt{T}} \sum_{i=1}^{n} \sum_{j=1}^{n} \sum_{t=1}^{T} \mu_{i t} \varepsilon_{j t} \\
= & \frac{1}{\sqrt{T}} \sum_{t=1}^{T}\left(\lambda_{t}^{2}-\sigma_{\lambda}^{2}\right)+O_{p}\left(T^{-1 / 4} n^{-\alpha / 4}\right)+O_{p}\left(n^{-1 / 2}\right)+O_{p}\left(n^{-\alpha / 2}\right),
\end{aligned}
$$

where the last equality follows from Lmma 1(b), 1(c) and 1(e) and the fact that $\frac{1}{\sqrt{T}} \sum_{t=1}^{T}\left(\frac{1}{n} \sum_{i=1}^{n} \mu_{i t}\right)^{2} \leq$ $\frac{1}{n} \sum_{i=1}^{n}\left(\sum_{t=1}^{T} \mu_{i t}^{4}\right)^{1 / 2}=O\left(n^{-\alpha / 2}\right)$. Then

$$
\sqrt{T}\left(\widehat{\sigma}_{\lambda}^{2}-\sigma_{\lambda}^{2}-\frac{1}{n^{2}} \sum_{i=1}^{n} \sigma_{\varepsilon i}^{2}\right) \rightarrow_{d} N\left(0, \Sigma_{\lambda}\right)
$$

as $(n, T \rightarrow \infty)$ by virtue of Lemma 1 (a). 
(b) Proof. Note that

$$
\begin{aligned}
& \sqrt{T}\left(\widehat{\sigma}_{\varepsilon i}^{2}-\left(\frac{n-1}{n}\right)^{2} \sigma_{\varepsilon i}^{2}-\frac{1}{n^{2}} \sum_{j \neq i}^{n} \sigma_{\varepsilon j}^{2}\right) \\
= & \frac{1}{\sqrt{T}} \sum_{t=1}^{T}\left(\mu_{i t}-\frac{1}{n} \sum_{i=1}^{n} \mu_{i t}\right)^{2}+\frac{2}{\sqrt{T}} \sum_{t=1}^{T}\left(\mu_{i t}-\frac{1}{n} \sum_{i=1}^{n} \mu_{i t}\right)\left(\varepsilon_{i t}-\frac{1}{n} \sum_{i=1}^{n} \varepsilon_{i t}\right) \\
& +\frac{1}{\sqrt{T}} \sum_{t=1}^{T}\left[\left(\varepsilon_{i t}-\frac{1}{n} \sum_{i=1}^{n} \varepsilon_{i t}\right)^{2}-\left(\frac{n-1}{n}\right)^{2} \sigma_{\varepsilon i}^{2}-\frac{1}{n^{2}} \sum_{j \neq i}^{n} \sigma_{\varepsilon j}^{2}\right] \\
= & \frac{1}{\sqrt{T}} \sum_{t=1}^{T}\left[\left(\varepsilon_{i t}-\frac{1}{n} \sum_{i=1}^{n} \varepsilon_{i t}\right)^{2}-\left(\frac{n-1}{n}\right)^{2} \sigma_{\varepsilon i}^{2}-\frac{1}{n^{2}} \sum_{j \neq i}^{n} \sigma_{\varepsilon j}^{2}\right] \\
& +O\left(n^{-\alpha / 2}\right)+O_{p}\left(T^{-1 / 4} n^{-\alpha / 4}\right)
\end{aligned}
$$

by virtue of Lemma 2(a) and 2(d). For each $i$,

$$
\begin{array}{rl} 
& \frac{1}{\sqrt{T}} \sum_{t=1}^{T}\left[\left(\varepsilon_{i t}-\frac{1}{n} \sum_{i=1}^{n} \varepsilon_{i t}\right)^{2}-\left(\frac{n-1}{n}\right)^{2} \sigma_{\varepsilon i}^{2}-\frac{1}{n^{2}} \sum_{j \neq i}^{n} \sigma_{\varepsilon j}^{2}\right] \\
= & \left(\frac{n-1}{n}\right)^{2} \frac{1}{\sqrt{T}} \sum_{t=1}^{T}\left(\varepsilon_{i t}^{2}-\sigma_{\varepsilon i}^{2}\right)-\frac{2(n-1)}{n^{2} \sqrt{T}} \sum_{t=1}^{T} \varepsilon_{i t} \sum_{j \neq i}^{n} \varepsilon_{j t} \\
& +\frac{1}{n \sqrt{T}} \sum_{t=1}^{T}\left[\left(\frac{1}{\sqrt{n}} \sum_{j \neq i}^{n} \varepsilon_{j t}\right)^{2}-\frac{1}{n} \sum_{j \neq i}^{n} \sigma_{\varepsilon j}^{2}\right] \\
= & \left(\frac{n-1}{n}\right)^{2} \frac{1}{\sqrt{T}} \sum_{t=1}^{T}\left(\varepsilon_{i t}^{2}-\sigma_{\varepsilon i}^{2}\right)+O_{p}\left(\frac{1}{\sqrt{n}}\right) \\
\rightarrow_{d} & N\left(0, \omega_{\varepsilon i}\right)
\end{array}
$$

in view of Lemma $1(\mathrm{~d})$ and1(e) because $\lim _{n \rightarrow \infty}\left(\frac{n-1}{n}\right)^{2}=1$. So the desired result follows immediately. 
(c) Using Lemma 4(b), we have

$$
\begin{aligned}
& \frac{1}{\sqrt{T}} \sum_{t=1}^{T}\left(\left(e_{i t}-\bar{e}_{\cdot t}\right)^{2}-\widehat{\sigma}_{\varepsilon i}^{2}\right)^{2} \\
= & \frac{1}{\sqrt{T}} \sum_{t=1}^{T}\left\{\left(e_{i t}-\bar{e}_{\cdot t}\right)^{2}-\left(\left(\frac{n-1}{n}\right)^{2} \sigma_{\varepsilon i}^{2}+\frac{1}{n^{2}} \sum_{j \neq i}^{n} \sigma_{\varepsilon j}^{2}\right)\right\}^{2} \\
& +O_{p}\left(T^{-1 / 2}\right) .
\end{aligned}
$$

But by Lemma 2(a)-2(e),

$$
\begin{aligned}
& \frac{1}{\sqrt{T}} \sum_{t=1}^{T}\left\{\left(e_{i t}-\bar{e}_{\cdot t}\right)^{2}-\left(\left(\frac{n-1}{n}\right)^{2} \sigma_{\varepsilon i}^{2}+\frac{1}{n^{2}} \sum_{j \neq i}^{n} \sigma_{\varepsilon j}^{2}\right)\right\}^{2} \\
= & \frac{1}{\sqrt{T}} \sum_{t=1}^{T}\left\{\left(\varepsilon_{i t}-\frac{1}{n} \sum_{i=1}^{n} \varepsilon_{i t}\right)^{2}-\left(\left(\frac{n-1}{n}\right)^{2} \sigma_{\varepsilon i}^{2}+\frac{1}{n^{2}} \sum_{j \neq i}^{n} \sigma_{\varepsilon j}^{2}\right)\right\}^{2} \\
& +O\left(T^{-1 / 2} n^{-\alpha}\right)+O_{p}\left(n^{-\alpha / 2}\right)+O_{p}\left(T^{-1 / 4} n^{-3 \alpha / 4}\right)+O_{p}\left(T^{-1 / 4} n^{-\alpha / 4}\right) .
\end{aligned}
$$

In addition, using Lemma $2(\mathrm{f})-2(\mathrm{~h})$, we can readily show that

$$
\begin{aligned}
& \frac{1}{\sqrt{T}} \sum_{t=1}^{T}\left\{\left(\varepsilon_{i t}-\frac{1}{n} \sum_{i=1}^{n} \varepsilon_{i t}\right)^{2}-\left(\left(\frac{n-1}{n}\right)^{2} \sigma_{\varepsilon i}^{2}+\frac{1}{n^{2}} \sum_{j \neq i}^{n} \sigma_{\varepsilon j}^{2}\right)\right\}^{2} \\
= & \frac{1}{\sqrt{T}} \sum_{t=1}^{T}\left(\frac{n-1}{n}\right)^{4}\left(\varepsilon_{i t}^{2}-\sigma_{\varepsilon i}^{2}\right)^{4}+\frac{1}{n^{2} \sqrt{T}} \sum_{t=1}^{T}\left[\left(\frac{1}{\sqrt{n}} \sum_{j \neq i}^{n} \varepsilon_{j t}\right)^{2}-\frac{1}{n} \sum_{j \neq i}^{n} \sigma_{\varepsilon j}^{2}\right]^{2} \\
& +\left(\frac{n-1}{n}\right)^{2} \frac{4}{n \sqrt{T}} \sum_{t=1}^{T} \varepsilon_{i t}^{2}\left(\frac{1}{\sqrt{n}} \sum_{j \neq i}^{n} \varepsilon_{j t}\right)^{2}+O_{p}\left(n^{-1 / 2}\right)
\end{aligned}
$$

Therefore, we have 


$$
\begin{aligned}
& \frac{1}{\sqrt{T}} \sum_{t=1}^{T}\left\{\left(\left(e_{i t}-\bar{e}_{. t}\right)^{2}-\widehat{\sigma}_{\varepsilon i}^{2}\right)^{2}-\left(\frac{n-1}{n}\right)^{4} \omega_{\varepsilon i}-\frac{1}{n^{2}} \xi_{n}-\frac{1}{n}\left(\frac{n-1}{n}\right)^{2} \sigma_{\varepsilon i}^{2}\left(\frac{1}{n} \sum_{j \neq i}^{n} \sigma_{\varepsilon j}^{2}\right)\right\} \\
= & \left(\frac{n-1}{n}\right)^{4} \frac{1}{\sqrt{T}} \sum_{t=1}^{T}\left\{\left(\varepsilon_{i t}^{2}-\sigma_{\varepsilon i}^{2}\right)^{2}-\omega_{\varepsilon i}\right\} \\
& +\frac{1}{n^{2}} \frac{1}{\sqrt{T}} \sum_{t=1}^{T}\left\{\left[\left(\frac{1}{\sqrt{n}} \sum_{j \neq i}^{n} \varepsilon_{j t}\right)^{2}-\frac{1}{n} \sum_{j \neq i}^{n} \sigma_{\varepsilon j}^{2}\right]^{2}-\xi_{n}\right\} \\
& +\frac{1}{n}\left(\frac{n-1}{n}\right)^{2} \frac{1}{\sqrt{T}} \sum_{t=1}^{T}\left\{\varepsilon_{i t}^{2}\left(\frac{1}{\sqrt{n}} \sum_{j \neq i}^{n} \varepsilon_{j t}\right)^{2}-\sigma_{\varepsilon i}^{2}\left(\frac{1}{n} \sum_{j \neq i}^{n} \sigma_{\varepsilon j}^{2}\right)\right\} \\
& +O_{p}\left(n^{-1 / 2}\right) \\
= & \left(\frac{n-1}{n}\right)^{4} \frac{1}{\sqrt{T}} \sum_{t=1}^{T}\left\{\left(\varepsilon_{i t}^{2}-\sigma_{\varepsilon i}^{2}\right)^{2}-\omega_{\varepsilon i}\right\}+O_{p}\left(n^{-1 / 2}\right)
\end{aligned}
$$

by virtue of Lemma $2(i)$ and $2(j)$. Since

$$
\frac{1}{\sqrt{T}} \sum_{t=1}^{T}\left\{\left(\varepsilon_{i t}^{2}-\sigma_{\varepsilon i}^{2}\right)^{2}-\omega_{\varepsilon i}\right\} \rightarrow_{d} N\left(0, \operatorname{var}\left(\left(\varepsilon_{i t}^{2}-\sigma_{\varepsilon i}^{2}\right)^{2}\right)\right)
$$

as $(n, T \rightarrow \infty)$ by classical central limit theorem for iid random variables $\left\{\left(\varepsilon_{i t}^{2}-\sigma_{\varepsilon i}^{2}\right)^{2}-\omega_{\varepsilon i}\right\}$, the desired result immediately follows.

Lemma 5. Suppose Assumptions 1-4 hold with $\widetilde{\sigma}_{\varepsilon i}^{2}=\left(\frac{n}{n-1}\right)^{2} \widehat{\sigma}_{\varepsilon i}^{2}-\left(\frac{n}{n-1}\right)^{2} \frac{1}{n^{2}} \sum_{j \neq i}^{n} \widehat{\sigma}_{\varepsilon j}^{2}, \widetilde{\sigma}_{\lambda}^{2}=$ $\widehat{\sigma}_{\lambda}^{2}-\frac{1}{n^{2}} \sum_{i=1}^{n} \widehat{\sigma}_{\varepsilon i}^{2}$, and $\widetilde{\omega}_{\varepsilon i T}=\left(\frac{n}{n-1}\right)^{4} \widehat{\omega}_{\varepsilon i T}-\frac{1}{n}\left(\frac{n}{n-1}\right)^{2} \widehat{\sigma}_{\varepsilon i}^{2}\left(\frac{1}{n} \sum_{j \neq i}^{n} \widehat{\sigma}_{\varepsilon j}^{2}\right)$. Then as $(n, T \rightarrow \infty)$,

(a) $\tilde{\sigma}_{\varepsilon i}^{2}-\sigma_{\varepsilon i}^{2}=O_{p}\left(n^{-2}\right)+O_{p}\left(T^{-1 / 2}\right)$,

(b) $\tilde{\sigma}_{\lambda}^{2}-\sigma_{\lambda}^{2}=O_{p}\left(n^{-2}\right)+O_{p}\left(T^{-1 / 2}\right)$,

(c) $\widetilde{\omega}_{\varepsilon i T}-\omega_{\varepsilon i}=O_{p}\left(n^{-2}\right)+O_{p}\left(T^{-1 / 2}\right)$.

Proof. The results follow immediately from Lemma 4 . 
Lemma 6. Suppose Assumptions 1-4 hold with $\widetilde{\Omega}_{i T}=\widetilde{\omega}_{\varepsilon i T}+4 \widetilde{\sigma}_{\lambda}^{2} \widetilde{\sigma}_{\varepsilon i}^{2}$ and $\Omega_{i}=\omega_{\varepsilon i}+4 \sigma_{\lambda}^{2} \sigma_{\varepsilon i}^{2}$ Then $\widetilde{\Omega}_{i T}-\Omega_{i}=O_{p}\left(T^{-1 / 2}\right)+O_{p}\left(n^{-2}\right)$.

Proof. The result follows readily from Lemma 5.

Theorem 1. Suppose Assumptions 1-4 hold. Then as $(n, T \rightarrow \infty)$,

(i) $\sqrt{T}\left(R M S E_{A F}^{2}-\sigma_{\lambda}^{2}-\frac{1}{n^{2}} \sum_{i=1}^{n} \sigma_{\varepsilon i}^{2}\right) \rightarrow_{d} N\left(0, \Sigma_{\lambda}\right)$.

(ii) $\sqrt{T}\left(R M S E_{R T}^{2}-\left(\frac{1}{n} \sum_{i=1}^{n} \sqrt{\sigma_{\lambda}^{2}+\sigma_{\varepsilon i}^{2}}\right)^{2}\right) \rightarrow_{d} N\left(0, \phi \Sigma_{\lambda}\right)$,

where $\phi=\left(\lim _{n \rightarrow \infty} \frac{1}{n} \sum_{i=1}^{n}\left(\sigma_{\lambda}^{2}+\sigma_{\varepsilon i}^{2}\right)^{1 / 2}\right)^{2}\left(\lim _{n \rightarrow \infty} \frac{1}{n} \sum_{i=1}^{n}\left(\sigma_{\lambda}^{2}+\sigma_{\varepsilon i}^{2}\right)^{-1 / 2}\right)^{2}$.

(iii) $\sqrt{T}\left(R M S E_{L P S}^{2}-\left(\sigma_{\lambda}^{2}+\frac{1}{n} \sum_{i=1}^{n} \sigma_{\varepsilon i}^{2}\right)\right) \rightarrow_{d} N\left(0, \Sigma_{\lambda}\right)$.

Proof.

(i) See the proof of Lemma 4(a).

(ii) Using Taylor's expansion and Lemma 1(b), we have

$$
\begin{aligned}
& T^{1 / 2}\left(R M S E_{R T}-\frac{1}{n} \sum_{i=1}^{n} \sqrt{\sigma_{\lambda}^{2}+\sigma_{\varepsilon i}^{2}}\right) \\
= & T^{1 / 2}\left\{\frac{1}{n} \sum_{i=1}^{n}\left(\sqrt{\frac{1}{T} \sum_{t=1}^{T} e_{i t}^{2}}-\sqrt{\sigma_{\lambda}^{2}+\sigma_{\varepsilon i}^{2}}\right)\right\} \\
= & \frac{1}{n} \sum_{i=1}^{n} \frac{1}{2}\left(\sigma_{\lambda}^{2}+\sigma_{\varepsilon i}^{2}\right)^{-1 / 2}\left\{\frac{1}{T^{1 / 2}} \sum_{t=1}^{T}\left[\left(\lambda_{t}+\varepsilon_{i t}\right)^{2}-\left(\sigma_{\lambda}^{2}+\sigma_{\varepsilon i}^{2}\right)\right]\right\} \\
& +O_{p}\left(n^{-\alpha / 2}\right)+O_{p}\left(T^{-1 / 4} n^{-\alpha / 4}\right) .
\end{aligned}
$$


for large $n$ and large $T$.

$$
\begin{aligned}
& \frac{1}{n} \sum_{i=1}^{n} \frac{1}{2}\left(\sigma_{\lambda}^{2}+\sigma_{\varepsilon i}^{2}\right)^{-1 / 2}\left\{\frac{1}{T^{1 / 2}} \sum_{t=1}^{T}\left[\left(\lambda_{t}+\varepsilon_{i t}\right)^{2}-\left(\sigma_{\lambda}^{2}+\sigma_{\varepsilon i}^{2}\right)\right]\right\} \\
= & \left(\frac{1}{2 n} \sum_{i=1}^{n}\left(\sigma_{\lambda}^{2}+\sigma_{\varepsilon i}^{2}\right)^{-1 / 2}\right) \frac{1}{T^{1 / 2}} \sum_{t=1}^{T}\left(\lambda_{t}^{2}-\sigma_{\lambda}^{2}\right) \\
& +\frac{1}{2 n} \sum_{i=1}^{n}\left(\sigma_{\lambda}^{2}+\sigma_{\varepsilon i}^{2}\right)^{-1 / 2} \frac{1}{T^{1 / 2}} \sum_{t=1}^{T}\left(\varepsilon_{i t}^{2}-\sigma_{\varepsilon i}^{2}\right) \\
& +\frac{1}{n} \sum_{i=1}^{n}\left(\sigma_{\lambda}^{2}+\sigma_{\varepsilon i}^{2}\right)^{-1 / 2} \frac{1}{T^{1 / 2}} \sum_{t=1}^{T} \lambda_{t} \varepsilon_{i t} \\
= & \left(\frac{1}{2 n} \sum_{i=1}^{n}\left(\sigma_{\lambda}^{2}+\sigma_{\varepsilon i}^{2}\right)^{-1 / 2}\right) \frac{1}{T^{1 / 2}} \sum_{t=1}^{T}\left(\lambda_{t}^{2}-\sigma_{\lambda}^{2}\right)+O_{p}\left(n^{-1 / 2}\right)
\end{aligned}
$$

because it can be easily shown that $\frac{1}{n^{1 / 2}} \sum_{i=1}^{n}\left(\sigma_{\lambda}^{2}+\sigma_{\varepsilon i}^{2}\right)^{-1 / 2} \frac{1}{T^{1 / 2}} \sum_{t=1}^{T}\left(\varepsilon_{i t}^{2}-\sigma_{\varepsilon i}^{2}\right)=O_{p}(1)$ and $\frac{1}{n^{1 / 2}} \sum_{i=1}^{n}\left(\sigma_{\lambda}^{2}+\sigma_{\varepsilon i}^{2}\right)^{-1 / 2} \frac{1}{T^{1 / 2}} \sum_{t=1}^{T} \lambda_{t} \varepsilon_{i t}=O_{p}(1)$ by using similar arguments as in the second part of Lemma 1(c). Then it follows that

$$
\begin{aligned}
T^{1 / 2}\left(R M S E_{R T}-\frac{1}{n} \sum_{i=1}^{n} \sqrt{\sigma_{\lambda}^{2}+\sigma_{\varepsilon i}^{2}}\right)= & \left(\frac{1}{2 n} \sum_{i=1}^{n}\left(\sigma_{\lambda}^{2}+\sigma_{\varepsilon i}^{2}\right)^{-1 / 2}\right) \frac{1}{T^{1 / 2}} \sum_{t=1}^{T}\left(\lambda_{t}^{2}-\sigma_{\lambda}^{2}\right) \\
& +O_{p}\left(n^{-1 / 2}\right)+O_{p}\left(n^{-\alpha / 2}\right)+O_{p}\left(T^{-1 / 4} n^{-\alpha / 4}\right) \\
\rightarrow & { }_{d} N\left(0, \frac{1}{4}\left(\lim _{n \rightarrow \infty} \frac{1}{n} \sum_{i=1}^{n}\left(\sigma_{\lambda}^{2}+\sigma_{\varepsilon i}^{2}\right)^{-1 / 2}\right)^{2} \Sigma_{\lambda}\right)
\end{aligned}
$$

as $(n, T \rightarrow \infty)$. Consequently,

$$
\begin{aligned}
& T^{1 / 2}\left(R M S E_{R T}^{2}-\left(\frac{1}{n} \sum_{i=1}^{n} \sqrt{\sigma_{\lambda}^{2}+\sigma_{\varepsilon i}^{2}}\right)^{2}\right) \\
= & \left(R M S E_{R T}+\frac{1}{n} \sum_{i=1}^{n} \sqrt{\sigma_{\lambda}^{2}+\sigma_{\varepsilon i}^{2}}\right) T^{1 / 2}\left(R M S E_{R T}-\frac{1}{n} \sum_{i=1}^{n} \sqrt{\sigma_{\lambda}^{2}+\sigma_{\varepsilon i}^{2}}\right) \\
\rightarrow & { }_{d} N\left(0,\left(\lim _{n \rightarrow \infty} \frac{1}{n} \sum_{i=1}^{n}\left(\sigma_{\lambda}^{2}+\sigma_{\varepsilon i}^{2}\right)^{1 / 2}\right)^{2}\left(\lim _{n \rightarrow \infty} \frac{1}{n} \sum_{i=1}^{n}\left(\sigma_{\lambda}^{2}+\sigma_{\varepsilon i}^{2}\right)^{-1 / 2}\right)^{2} \Sigma_{\lambda}\right),
\end{aligned}
$$


as $(n, T \rightarrow \infty)$ since $R M S E_{R T}+\frac{1}{n} \sum_{i=1}^{n} \sqrt{\sigma_{\lambda}^{2}+\sigma_{\varepsilon i}^{2}}=\frac{2}{n} \sum_{i=1}^{n} \sqrt{\sigma_{\lambda}^{2}+\sigma_{\varepsilon i}^{2}}+O_{p}\left(T^{-1 / 2}\right)$.

(iii) Write

$$
\begin{aligned}
& T^{1 / 2}\left(R M S E_{L P S}^{2}-\left(\sigma_{\lambda}^{2}+\frac{1}{n} \sum_{i=1}^{n} \sigma_{\varepsilon i}^{2}\right)\right) \\
= & \frac{1}{T^{1 / 2}} \sum_{t=1}^{T}\left(\lambda_{t}^{2}-\sigma_{\lambda}^{2}\right)+\frac{1}{n T^{1 / 2}} \sum_{i=1}^{n} \sum_{t=1}^{T}\left(\varepsilon_{i t}^{2}-\sigma_{\varepsilon i}^{2}\right)+\frac{2}{n T^{1 / 2}} \sum_{i=1}^{n} \sum_{t=1}^{T} \lambda_{t} \varepsilon_{i t} \\
& +\frac{1}{n T^{1 / 2}} \sum_{i=1}^{n} \sum_{t=1}^{T} \mu_{i t}^{2}+\frac{2}{n T^{1 / 2}} \sum_{i=1}^{n} \sum_{t=1}^{T} \mu_{i t} \lambda_{t}+\frac{1}{n T^{1 / 2}} \sum_{i=1}^{n} \sum_{t=1}^{T} \mu_{i t} \varepsilon_{i t} \\
= & \frac{1}{T^{1 / 2}} \sum_{t=1}^{T}\left(\lambda_{t}^{2}-\sigma_{\lambda}^{2}\right)+O_{p}\left(n^{-1 / 2}\right)+O_{p}\left(n^{-\alpha / 2}\right)+O_{p}\left(T^{-1 / 4} n^{-\alpha / 4}\right),
\end{aligned}
$$

by virtue of Lemma $1(\mathrm{~b})-1(\mathrm{~d})$ and the fact that $\frac{1}{n T^{1 / 2}} \sum_{i=1}^{n} \sum_{t=1}^{T} \mu_{i t}^{2}=O_{p}\left(n^{-\alpha / 2}\right)$. Hence

$$
T^{1 / 2}\left(R M S E_{L P S}^{2}-\left(\sigma_{\lambda}^{2}+\frac{1}{n} \sum_{i=1}^{n} \sigma_{\varepsilon i}^{2}\right)\right) \rightarrow_{d} N\left(0, \Sigma_{\lambda}\right)
$$

holds as $(n, T \rightarrow \infty)$ in view of Lemma $1(\mathrm{a})$.

Corollary 1. Suppose Assumptions 1-4 hold. Then as $(n, T \rightarrow \infty)$,

(i) $R M S E_{A F}^{2} \rightarrow{ }_{p} \sigma_{\lambda}^{2}$.

(ii) $R M S E_{R T}^{2} \rightarrow_{p}\left(\lim _{n \rightarrow \infty} \frac{1}{n} \sum_{i=1}^{n} \sqrt{\sigma_{\lambda}^{2}+\sigma_{\varepsilon i}^{2}}\right)^{2}$.

(iii) $R M S E_{L P S}^{2} \rightarrow_{p}\left(\sigma_{\lambda}^{2}+\lim _{n \rightarrow \infty} \frac{1}{n} \sum_{i=1}^{n} \sigma_{\varepsilon i}^{2}\right)$.

Proof. The results follow immediately from Theorem 1.

Theorem 2. Suppose Assumptions 1-4 hold and $\widetilde{\omega}_{\varepsilon}=\frac{1}{n} \sum_{i=1}^{n} \widetilde{\omega}_{\varepsilon i T}$. Then under the null hypothesis that $\frac{1}{n} \sum_{i=1}^{n} \sigma_{\varepsilon i}^{2}=o\left(T^{-1 / 2} n^{-1 / 2}\right)$,

$$
Z=\sqrt{n T} \widetilde{\omega}_{\varepsilon}^{-1 / 2}\left\{R M S E_{L P S}^{2}-R M S E_{A F}^{2}\right\} \rightarrow_{d} N(0,1)
$$


as $(n, T \rightarrow \infty)$ and $\frac{n}{T} \rightarrow 0$.

Proof. Note that

$$
\begin{aligned}
\sqrt{n T}\left(R M S E_{L P S}^{2}-R M S E_{A F}^{2}\right)= & \sqrt{n T}\left\{\frac{1}{n T} \sum_{t=1}^{T} \sum_{i=1}^{n}\left(e_{i t}-\left(\frac{1}{n} \sum_{i=1}^{n} e_{i t}\right)\right)^{2}\right\} \\
= & \frac{1}{\sqrt{n T}} \sum_{t=1}^{T} \sum_{i=1}^{n} \mu_{i t}^{2}-\frac{1}{\sqrt{n T}} \sum_{t=1}^{T}\left(\frac{1}{n} \sum_{i=1}^{n} \mu_{i t}\right)^{2} \\
& +\frac{1}{\sqrt{n T}} \sum_{t=1}^{T} \sum_{i=1}^{n} \varepsilon_{i t}^{2}-\frac{1}{\sqrt{n T}} \sum_{t=1}^{T}\left(\frac{1}{n} \sum_{i=1}^{n} \varepsilon_{i t}\right)^{2} \\
& +\frac{2}{\sqrt{n T}} \sum_{t=1}^{T} \sum_{i=1}^{n} \mu_{i t} \varepsilon_{i t}-\frac{2}{\sqrt{n T}} \sum_{t=1}^{T}\left(\frac{1}{n} \sum_{i=1}^{n} \mu_{i t}\right)\left(\frac{1}{n} \sum_{i=1}^{n} \varepsilon_{i t}\right) \\
= & \frac{1}{\sqrt{n T}} \sum_{t=1}^{T} \sum_{i=1}^{n} \varepsilon_{i t}^{2}-\frac{1}{n \sqrt{n T}} \sum_{t=1}^{T}\left(\frac{1}{\sqrt{n}} \sum_{i=1}^{n} \varepsilon_{i t}\right)^{2} \\
& +O_{p}\left(n^{(1-\alpha) / 2}\right)+O_{p}\left(T-1 / 4 n^{(2-\alpha) / 4}\right)
\end{aligned}
$$

by virtue of Lemma $1(\mathrm{~b})$ and the following results

$$
\frac{1}{T} \sum_{t=1}^{T}\left(\frac{1}{n} \sum_{i=1}^{n} \mu_{i t}\right)^{2} \leq \frac{1}{n T} \sum_{t=1}^{T} \sum_{i=1}^{n} \mu_{i t}^{2} \leq \frac{1}{n} \sum_{i=1}^{n}\left(\frac{1}{T} \sum_{t=1}^{T} \mu_{i t}^{4}\right)^{1 / 2}=O_{p}\left(T^{-1 / 2} n^{-\alpha / 2}\right)
$$

and

$$
\frac{2}{\sqrt{T}} \sum_{t=1}^{T}\left(\frac{1}{n} \sum_{i=1}^{n} \mu_{i t}\right)\left(\frac{1}{n} \sum_{i=1}^{n} \varepsilon_{i t}\right)=\frac{1}{n^{2}} \sum_{i=1}^{n} \sum_{j=1}^{n} \frac{2}{\sqrt{T}} \sum_{t=1}^{T} \mu_{i t} \varepsilon_{j t}=O_{p}\left(T^{-1 / 4} n^{-\alpha / 4}\right) .
$$


Therefore,

$$
\begin{aligned}
& \sqrt{n T}\left(R M S E_{L P S}^{2}-R M S E_{A F}^{2}\right) \\
= & \frac{1}{\sqrt{n T}} \sum_{i=1}^{n} \sum_{t=1}^{T}\left(\varepsilon_{i t}^{2}-\sigma_{\varepsilon i}^{2}\right)-\frac{1}{n^{3 / 2} \sqrt{T}} \sum_{t=1}^{T}\left[\left(\frac{1}{\sqrt{n}} \sum_{i=1}^{n} \varepsilon_{i t}\right)^{2}-\frac{1}{n} \sum_{i=1}^{n} \sigma_{\varepsilon i}^{2}\right] \\
& +\left(1-\frac{1}{n^{2}}\right) \frac{\sqrt{n T}}{n} \sum_{i=1}^{n} \sigma_{\varepsilon i}^{2}+O_{p}\left(n^{(1-\alpha) / 2}\right)+O_{p}\left(T^{-1 / 4} n^{(2-\alpha) / 4}\right) \\
= & \frac{1}{\sqrt{n T}} \sum_{i=1}^{n} \sum_{t=1}^{T}\left(\varepsilon_{i t}^{2}-\sigma_{\varepsilon i}^{2}\right)+\left(1-\frac{1}{n^{2}}\right) \frac{\sqrt{n T}}{n} \sum_{i=1}^{n} \sigma_{\varepsilon i}^{2} \\
& +O_{p}\left(n^{(1-\alpha) / 2}\right)+O_{p}\left(n^{-3 / 2}\right)+O_{p}\left(T^{-1 / 4} n^{(2-\alpha) / 4}\right) .
\end{aligned}
$$

Under the null hypothesis, $\left(1-\frac{1}{n^{2}}\right) \frac{\sqrt{n T}}{n} \sum_{i=1}^{n} \sigma_{\varepsilon i}^{2}=o(1)$ as $(n, T \rightarrow \infty)$. Hence

$$
\sqrt{n T}\left(R M S E_{L P S}^{2}-R M S E_{A F}^{2}\right) \rightarrow_{d} N\left(0, \omega_{\varepsilon}\right)
$$

by Lemma $1(\mathrm{~d})$ provided $(n, T \rightarrow \infty)$ and $\frac{n^{2-\alpha}}{T} \rightarrow 0$, where the latter holds because $\frac{n^{2-\alpha}}{T}<\frac{n}{T}$ for $\alpha>1$ and $\frac{n}{T} \rightarrow 0$ by assumption. Next, since $\widetilde{\omega}_{\varepsilon}=\frac{1}{n} \sum_{i=1}^{n} \widetilde{\omega}_{\varepsilon i T}$ and $\widetilde{\omega}_{\varepsilon i T}-\omega_{\varepsilon i}=O_{p}\left(T^{-1 / 2}\right)+$ $O_{p}\left(n^{-2}\right)$ in view of Lemma $5(\mathrm{c})$, we see that

$$
\widetilde{\omega}_{\varepsilon}=\frac{1}{n} \sum_{i=1}^{n} \widetilde{\omega}_{\varepsilon i T} \rightarrow_{p} \omega_{\varepsilon}
$$

as $(n, T \rightarrow \infty)$. Consequently, under the null hypothesis,

$$
Z=\sqrt{n T} \widetilde{\omega}_{\varepsilon}^{-1 / 2}\left\{R M S E_{L P S}^{2}-R M S E_{A F}^{2}\right\} \rightarrow_{d} N(0,1)
$$

provided $(n, T \rightarrow \infty)$ and $\frac{n}{T} \rightarrow 0$.

Theorem 3. Suppose Assumptions 1-4 hold. Then under the null hypothesis that 


$$
\begin{aligned}
\frac{1}{n} \sum_{i=1}^{n}\left(\sigma_{\varepsilon i}^{2}-\frac{1}{n} \sum_{i=1}^{n} \sigma_{\varepsilon i}^{2}\right)^{2} & =o\left(T^{-1} n^{-1}\right), \\
W & =\frac{Y_{n T}^{\widehat{\beta}}-\widehat{Y}_{n T}^{\widehat{\beta}}-\frac{1}{2} \widehat{\beta}(\widehat{\beta}-1) \widehat{Y}_{n T}^{\widehat{\beta}-2}}{\widehat{\beta} \widehat{Y}_{n T}^{\widehat{\beta}-1}} \rightarrow_{d} N(0,1),
\end{aligned}
$$

as $(n, T \rightarrow \infty)$ and $\frac{n}{T} \rightarrow 0$, where $Y_{n T}=\frac{1}{s_{n T}} \sum_{i=1}^{n}\left[T\left(\frac{1}{T} \sum_{t=1}^{T} e_{i t}^{2}-\frac{1}{n T} \sum_{i=1}^{n} \sum_{t=1}^{T} e_{i t}^{2}\right)^{2}\right], \widehat{Y}_{n T}=$ $\frac{1}{s_{n T}} \sum_{i=1}^{n}\left(\frac{n-1}{n}\right)^{2} \widetilde{\Omega}_{i T}$ and $\widehat{\beta}=1-\frac{2}{3} \frac{\left(\sum_{i=1}^{n} \widetilde{\Omega}_{i T}\right)\left(\sum_{i=1}^{n} \widetilde{\Omega}_{i T}^{3}\right)}{\left(\sum_{i=1}^{n} \widetilde{\Omega}_{i T}^{2}\right)}$ with $s_{n T}^{2}=2 \sum_{i=1}^{n}\left(\frac{n-1}{n}\right)^{2} \widetilde{\Omega}_{i T}$.

Proof. Observe that if $Y_{n T}-\widehat{Y}_{n T} \rightarrow_{d} N(0,1)$ as $(n, T \rightarrow \infty)$ and $\frac{n}{T} \rightarrow 0$, then it follows by Taylor's expansion that

$$
W=\frac{Y_{n T}^{\widehat{\beta}}-\widehat{Y}_{n T}^{\widehat{\beta}}-\frac{1}{2} \widehat{\beta}(\widehat{\beta}-1) \widehat{Y}_{n T}^{\widehat{\beta}-2}}{\widehat{\beta} \widehat{Y}_{n T}^{\widehat{\beta}-1}} \rightarrow_{d} N(0,1)
$$

as $(n, T \rightarrow \infty)$ and $\frac{n}{T} \rightarrow 0$ since $\widehat{Y}_{n T}^{-1}=\frac{1}{s_{n T}} \sum_{i=1}^{n}\left(\frac{n-1}{n}\right)^{2} \widetilde{\Omega}_{i T}=O_{p}\left(n^{-1 / 2}\right)$ and $\widehat{\beta} \rightarrow_{p} 1-$ $\frac{2}{3} \frac{\left(\lim _{n \rightarrow \infty} \frac{1}{n} \sum_{i=1}^{n} \Omega_{i}\right)\left(\lim _{n \rightarrow \infty} \frac{1}{n} \sum_{i=1}^{n} \Omega_{i}^{3}\right)}{\left(\lim _{n \rightarrow \infty} \frac{1}{n} \sum_{i=1}^{n} \Omega_{i}^{2}\right)^{2}}$ by virtue of Lemma 6 . Thus it suffices to establish

$$
Y_{n T}-\widehat{Y}_{n T} \rightarrow_{d} N(0,1)
$$

or,

$$
\frac{1}{s_{n T}} \sum_{i=1}^{n}\left\{\left[T\left(\frac{1}{T} \sum_{t=1}^{T} e_{i t}^{2}-\frac{1}{n T} \sum_{i=1}^{n} \sum_{t=1}^{T} e_{i t}^{2}\right)^{2}\right]-\left(\frac{n-1}{n}\right)^{2} \widetilde{\Omega}_{i T}\right\} \rightarrow_{d} N(0,1)
$$

as $(n, T \rightarrow \infty)$ and $\frac{n}{T} \rightarrow 0$. Now write 


$$
\begin{aligned}
& \frac{1}{n^{1 / 2}} \sum_{i=1}^{n}\left(\frac{1}{T^{1 / 2}} \sum_{t=1}^{T}\left(e_{i t}^{2}-\frac{1}{n} \sum_{i=1}^{n} e_{i t}^{2}\right)\right)^{2} \\
= & \frac{1}{n^{1 / 2}} \sum_{i=1}^{n}\left(\frac{1}{T^{1 / 2}} \sum_{t=1}^{T}\left(\left(e_{i t}^{2}-E e_{i t}^{2}\right)-\frac{1}{n} \sum_{i=1}^{n}\left(e_{i t}^{2}-E e_{i t}^{2}\right)\right)\right)^{2} \\
& +\frac{1}{n^{1 / 2}} \sum_{i=1}^{n}\left(\frac{1}{T^{1 / 2}} \sum_{t=1}^{T} E e_{i t}^{2}-\frac{1}{n T^{1 / 2}} \sum_{i=1}^{n} \sum_{t=1}^{T} E e_{i t}^{2}\right)^{2} \\
& +\frac{2}{n^{1 / 2}} \sum_{i=1}^{n}\left(\frac{1}{T^{1 / 2}} \sum_{t=1}^{T}\left(\left(e_{i t}^{2}-E e_{i t}^{2}\right)-\frac{1}{n} \sum_{i=1}^{n}\left(e_{i t}^{2}-E e_{i t}^{2}\right)\right)\right)\left(\frac{1}{T^{1 / 2}} \sum_{t=1}^{T} E e_{i t}^{2}-\frac{1}{n T^{1 / 2}} \sum_{i=1}^{n} \sum_{t=1}^{T} E e_{i t}^{2}\right) .
\end{aligned}
$$

Expanding the first term on the right hand side of (17) yields

$$
\begin{aligned}
& \frac{1}{n^{1 / 2}} \sum_{i=1}^{n}\left(\frac{1}{T^{1 / 2}} \sum_{t=1}^{T}\left(\left(e_{i t}^{2}-E e_{i t}^{2}\right)-\frac{1}{n} \sum_{i=1}^{n}\left(e_{i t}^{2}-E e_{i t}^{2}\right)\right)\right)^{2} \\
& =\frac{1}{n^{1 / 2}} \sum_{i=1}^{n}\left(\frac{n-1}{n} \frac{1}{\sqrt{T}} \sum_{t=1}^{T}\left[\left(\varepsilon_{i t}^{2}-\sigma_{\varepsilon i}^{2}\right)+2 \lambda_{t} \varepsilon_{i t}\right]\right)^{2} \\
& -\frac{2}{n^{1 / 2}} \sum_{i=1}^{n}\left(\frac{n-1}{n} \frac{1}{\sqrt{T}} \sum_{t=1}^{T}\left[\left(\varepsilon_{i t}^{2}-\sigma_{\varepsilon i}^{2}\right)+2 \lambda_{t} \varepsilon_{i t}\right]\right)\left(\frac{1}{n \sqrt{T}} \sum_{j \neq i}^{n} \sum_{t=1}^{T}\left[\left(\varepsilon_{j t}^{2}-\sigma_{\varepsilon j}^{2}\right)+2 \lambda_{t} \varepsilon_{j t}\right]\right) \\
& +\frac{1}{n^{1 / 2}} \sum_{i=1}^{n}\left(\frac{1}{n \sqrt{T}} \sum_{j \neq i}^{n} \sum_{t=1}^{T}\left[\left(\varepsilon_{j t}^{2}-\sigma_{\varepsilon j}^{2}\right)+2 \lambda_{t} \varepsilon_{j t}\right]\right)^{2} \\
& +\frac{4}{n^{1 / 2}} \sum_{i=1}^{n}\left(\frac{1}{\sqrt{T}} \sum_{t=1}^{T}\left[\lambda_{t} \mu_{i t}+\mu_{i t} \varepsilon_{i t}\right]\right)^{2}-4 n^{1 / 2}\left(\frac{1}{n \sqrt{T}} \sum_{i=1}^{n} \sum_{t=1}^{T}\left[\lambda_{t} \mu_{i t}+\mu_{i t} \varepsilon_{i t}\right]\right)^{2} \\
& +\frac{4}{n^{1 / 2}} \sum_{i=1}^{n}\left(\frac{1}{\sqrt{T}} \sum_{t=1}^{T}\left[\left(\varepsilon_{i t}^{2}-\sigma_{\varepsilon i}^{2}\right)+2 \lambda_{t} \varepsilon_{i t}\right]\right)\left(\frac{1}{\sqrt{T}} \sum_{t=1}^{T}\left[\lambda_{t} \mu_{i t}+\mu_{i t} \varepsilon_{i t}\right]\right) \\
& -4 n^{1 / 2}\left(\frac{1}{n \sqrt{T}} \sum_{i=1}^{n} \sum_{t=1}^{T}\left[\lambda_{t} \mu_{i t}+\mu_{i t} \varepsilon_{i t}\right]\right)\left(\frac{1}{n \sqrt{T}} \sum_{i=1}^{n} \sum_{t=1}^{T}\left[\left(\varepsilon_{i t}^{2}-\sigma_{\varepsilon i}^{2}\right)+2 \lambda_{t} \varepsilon_{i t}\right]\right) \\
& =\frac{1}{n^{1 / 2}} \sum_{i=1}^{n}\left(\frac{n-1}{n} \frac{1}{\sqrt{T}} \sum_{t=1}^{T}\left[\left(\varepsilon_{i t}^{2}-\sigma_{\varepsilon i}^{2}\right)+2 \lambda_{t} \varepsilon_{i t}\right]\right)^{2} \\
& +O_{p}\left(T^{-1 / 2} n^{(1-\alpha) / 2}\right)+O_{p}\left(T^{-1 / 4} n^{(2-\alpha) / 4}\right)+O_{p}\left(T^{-1 / 4} n^{-\alpha / 4}\right)+O_{p}\left(n^{-1 / 2}\right)
\end{aligned}
$$


by virtue of Assumption 1, Lemma 1(b), 1(e), 1(f) and 1(g). Thus

$$
\begin{aligned}
& \frac{1}{n^{1 / 2}} \sum_{i=1}^{n}\left(\frac{1}{T^{1 / 2}} \sum_{t=1}^{T}\left(\left(e_{i t}^{2}-E e_{i t}^{2}\right)-\frac{1}{n} \sum_{i=1}^{n}\left(e_{i t}^{2}-E e_{i t}^{2}\right)\right)\right)^{2} \\
= & \frac{1}{n^{1 / 2}} \sum_{i=1}^{n}\left(\frac{n-1}{n} \frac{1}{\sqrt{T}} \sum_{t=1}^{T}\left[\left(\varepsilon_{i t}^{2}-\sigma_{\varepsilon i}^{2}\right)+2 \lambda_{t} \varepsilon_{i t}\right]\right)^{2}+o_{p}(1)
\end{aligned}
$$

provided $(n, T \rightarrow \infty)$ and $\frac{n^{2-\alpha}}{T} \rightarrow 0$, where the latter holds given $\frac{n}{T} \rightarrow 0$ and $\alpha>1$. But

$$
\frac{1}{\sqrt{2 \sum_{i=1}^{n} \Omega_{i}}} \sum_{i=1}^{n}\left\{\left(\frac{1}{\sqrt{T}} \sum_{t=1}^{T}\left[\left(\varepsilon_{i t}^{2}-\sigma_{\varepsilon i}^{2}\right)+2 \lambda_{t} \varepsilon_{i t}\right]\right)^{2}-\Omega_{i}\right\} \rightarrow_{d} N(0,1)
$$

holds as $(n, T \rightarrow \infty)$ by Theorem 2 of Phillips and Moon (1999). It follows immediately that

$$
\frac{1}{n^{1 / 2}} \sum_{i=1}^{n}\left(\frac{1}{T^{1 / 2}} \sum_{t=1}^{T}\left(\left(e_{i t}^{2}-E e_{i t}^{2}\right)-\frac{1}{n} \sum_{i=1}^{n}\left(e_{i t}^{2}-E e_{i t}^{2}\right)\right)\right)^{2}=O_{p}\left(n^{1 / 2}\right)
$$

because $0<\inf _{i} \Omega_{i} \leq \sup _{i} \Omega_{i}<\infty$. For the second term on the right hand side of (17), observe that

$$
\begin{aligned}
& \frac{1}{n^{1 / 2}} \sum_{i=1}^{n}\left(\frac{1}{T^{1 / 2}} \sum_{t=1}^{T} E e_{i t}^{2}-\frac{1}{n T^{1 / 2}} \sum_{i=1}^{n} \sum_{t=1}^{T} E e_{i t}^{2}\right)^{2} \\
= & \frac{1}{n^{1 / 2}} \sum_{i=1}^{n}\left(\frac{1}{T^{1 / 2}} \sum_{t=1}^{T}\left(\mu_{i t}^{2}-\frac{1}{n} \sum_{i=1}^{n} \mu_{i t}^{2}\right)\right)^{2}+\frac{T}{n^{1 / 2}} \sum_{i=1}^{n}\left(\sigma_{\varepsilon i}^{2}-\frac{1}{n} \sum_{i=1}^{n} \sigma_{\varepsilon i}^{2}\right)^{2} \\
& +\frac{2}{n^{1 / 2}} \sum_{i=1}^{n}\left(\frac{1}{T^{1 / 2}} \sum_{t=1}^{T}\left(\mu_{i t}^{2}-\frac{1}{n} \sum_{i=1}^{n} \mu_{i t}^{2}\right)\right)\left(T^{1 / 2}\left(\sigma_{\varepsilon i}^{2}-\frac{1}{n} \sum_{i=1}^{n} \sigma_{\varepsilon i}^{2}\right)\right) .
\end{aligned}
$$

Using Jensen inequality (repeatedly) and the inequality $2 a b \leq a^{2}+b^{2}$, we see that

$$
\frac{1}{n^{1 / 2}} \sum_{i=1}^{n}\left(\frac{1}{T^{1 / 2}} \sum_{t=1}^{T}\left(\mu_{i t}^{2}-\frac{1}{n} \sum_{i=1}^{n} \mu_{i t}^{2}\right)\right)^{2}=O\left(n^{\frac{1-2 \alpha}{2}}\right)
$$


Also, under the null hypothesis, we have

$$
\frac{T}{n^{1 / 2}} \sum_{i=1}^{n}\left(\sigma_{\varepsilon i}^{2}-\frac{1}{n} \sum_{i=1}^{n} \sigma_{\varepsilon i}^{2}\right)^{2}=o\left(n^{-1 / 2}\right)
$$

and hence

$$
\begin{aligned}
& \frac{2}{n^{1 / 2}} \sum_{i=1}^{n}\left(\frac{1}{T^{1 / 2}} \sum_{t=1}^{T}\left(\mu_{i t}^{2}-\frac{1}{n} \sum_{i=1}^{n} \mu_{i t}^{2}\right)\right)\left(T^{1 / 2}\left(\sigma_{\varepsilon i}^{2}-\frac{1}{n} \sum_{i=1}^{n} \sigma_{\varepsilon i}^{2}\right)\right) \\
\leq & 2\left\{\frac{1}{n^{1 / 2}} \sum_{i=1}^{n}\left[\frac{1}{T^{1 / 2}} \sum_{t=1}^{T}\left(\mu_{i t}^{2}-\frac{1}{n} \sum_{i=1}^{n} \mu_{i t}^{2}\right)\right]^{2}\right\}^{1 / 2}\left\{\frac{T}{n^{1 / 2}} \sum_{i=1}^{n}\left(\sigma_{\varepsilon i}^{2}-\frac{1}{n} \sum_{i=1}^{n} \sigma_{\varepsilon i}^{2}\right)^{2}\right\}^{1 / 2} \\
= & O\left(n^{\frac{1-2 \alpha}{4}}\right) o\left(n^{-1 / 4}\right) \\
= & o\left(n^{-\alpha / 2}\right)
\end{aligned}
$$

by means of Cauchy Schwarz inequality. Thus under the null hypothesis and for $\alpha>1$, we have

$$
\frac{1}{n^{1 / 2}} \sum_{i=1}^{n}\left(\frac{1}{T^{1 / 2}} \sum_{t=1}^{T} E e_{i t}^{2}-\frac{1}{n T^{1 / 2}} \sum_{i=1}^{n} \sum_{t=1}^{T} E e_{i t}^{2}\right)^{2}=o\left(n^{-1 / 2}\right),
$$

from which we can, via Cauchy Schwarz inequality again, obtain the order of the third term on the right hand side of (17),

$$
\begin{aligned}
& \frac{2}{n^{1 / 2}} \sum_{i=1}^{n}\left(\frac{1}{T^{1 / 2}} \sum_{t=1}^{T}\left(\left(e_{i t}^{2}-E e_{i t}^{2}\right)-\frac{1}{n} \sum_{i=1}^{n}\left(e_{i t}^{2}-E e_{i t}^{2}\right)\right)\right)\left(\frac{1}{T^{1 / 2}} \sum_{t=1}^{T} E e_{i t}^{2}-\frac{1}{n T^{1 / 2}} \sum_{i=1}^{n} \sum_{t=1}^{T} E e_{i t}^{2}\right) \\
\leq & 2\left\{\frac{1}{n^{1 / 2}} \sum_{i=1}^{n}\left[\frac{1}{T^{1 / 2}} \sum_{t=1}^{T}\left(\left(e_{i t}^{2}-E e_{i t}^{2}\right)-\frac{1}{n} \sum_{i=1}^{n}\left(e_{i t}^{2}-E e_{i t}^{2}\right)\right)\right]^{2}\right\}^{1 / 2} \\
& \times\left\{\frac{1}{n^{1 / 2}} \sum_{i=1}^{n}\left[\frac{1}{T^{1 / 2}} \sum_{t=1}^{T} E e_{i t}^{2}-\frac{1}{n T^{1 / 2}} \sum_{i=1}^{n} \sum_{t=1}^{T} E e_{i t}^{2}\right]^{2}\right\}^{1 / 2} \\
= & O_{p}\left(n^{1 / 4}\right) o_{p}\left(n^{-1 / 4}\right) \\
= & o_{p}(1)
\end{aligned}
$$


Combining (18), (19) and (20) then yields

$$
\begin{aligned}
& \frac{1}{\sqrt{n}} \sum_{i=1}^{n}\left\{\left[T\left(\frac{1}{T} \sum_{t=1}^{T} e_{i t}^{2}-\frac{1}{n T} \sum_{i=1}^{n} \sum_{t=1}^{T} e_{i t}^{2}\right)^{2}\right]-\left(\frac{n-1}{n}\right)^{2} \widetilde{\Omega}_{i T}\right\} \\
= & \frac{1}{\sqrt{n}} \sum_{i=1}^{n}\left\{\left[\frac{n-1}{n} \frac{1}{\sqrt{T}} \sum_{t=1}^{T}\left[\left(\varepsilon_{i t}^{2}-\sigma_{\varepsilon i}^{2}\right)+2 \lambda_{t} \varepsilon_{i t}\right]\right]^{2}-\left(\frac{n-1}{n}\right)^{2} \widetilde{\Omega}_{i T}\right\} \\
= & \left(\frac{n-1}{n}\right)^{2} \frac{1}{\sqrt{n}} \sum_{i=1}^{n}\left\{\left[\frac{1}{\sqrt{T}} \sum_{t=1}^{T}\left[\left(\varepsilon_{i t}^{2}-\sigma_{\varepsilon i}^{2}\right)+2 \lambda_{t} \varepsilon_{i t}\right]\right]^{2}-\Omega_{i}\right\}+o_{p}(1),
\end{aligned}
$$

by virtue of Lemma 6 provided $(n, T \rightarrow \infty)$ and $\frac{n}{T} \rightarrow 0$, which, together with the following result (implied also by Lemma 6)

$$
\frac{s_{n T}}{\sqrt{n}}=2^{1 / 2}\left(\frac{n-1}{n}\right) \sqrt{\frac{1}{n} \sum_{i=1}^{n} \Omega_{i}}+O_{p}\left(T^{-1 / 2}\right)+O_{p}\left(n^{-2}\right),
$$

gives rise to

$$
\begin{aligned}
& \frac{1}{s_{n T}} \sum_{i=1}^{n}\left\{\left[T\left(\frac{1}{T} \sum_{t=1}^{T} e_{i t}^{2}-\frac{1}{n T} \sum_{i=1}^{n} \sum_{t=1}^{T} e_{i t}^{2}\right)^{2}\right]-\left(\frac{n-1}{n}\right)^{2} \widetilde{\Omega}_{i T}\right\} \\
= & \left(\frac{n-1}{n}\right) \frac{1}{2^{1 / 2} \sqrt{\sum_{i=1}^{n} \Omega_{i}}} \sum_{i=1}^{n}\left\{\left[\frac{1}{\sqrt{T}} \sum_{t=1}^{T}\left[\left(\varepsilon_{i t}^{2}-\sigma_{\varepsilon i}^{2}\right)+2 \lambda_{t} \varepsilon_{i t}\right]\right]^{2}-\Omega_{i}\right\}+o_{p}(1)
\end{aligned}
$$

Thus, we conclude that

$$
\frac{1}{s_{n T}} \sum_{i=1}^{n}\left\{\left[T\left(\frac{1}{T} \sum_{t=1}^{T} e_{i t}^{2}-\frac{1}{n T} \sum_{i=1}^{n} \sum_{t=1}^{T} e_{i t}^{2}\right)^{2}\right]-\left(\frac{n-1}{n}\right)^{2} \widetilde{\Omega}_{i T}\right\} \rightarrow_{d} N(0,1)
$$

as $(n, T \rightarrow \infty)$ and $\frac{n}{T} \rightarrow 0$ by Theorem 2 of Phillips and Moon (1999). 


\section{References}

Baker, S.R., N. Bloom and S.J. Davis (2013). Measuring economic policy uncertainty. Stanford Department of Economics Working paper.

Baltagi, B.H., G. Bresson and A. Pirotte (2006). Joint LM test for heteroskedasticity in a one way error component model. Journal of Econometrics 134, 401-417.

Bates, J.M. and C.W.J. Granger (1969). The combination of forecasts. Operational Research Quarterly 20, 451-468.

Bloom, N. (2009). The impact of uncertainty shocks. Econometrica 77, 623-685.

Buckland, S.T., K.P. Burnham, and N.H. Augustin (1997). Model selection: an integral part of inference. Biometrics 53, 603-618.

Chen, W.W. and R.S. Deo (2004). Power transformations to induce normality and their applications. Journal of the Royal Statistical Society B 66, 117-130.

Clemen, R.T. (1989). Combining forecasts: a review and annotated bibliography. International Journal of Forecasting 5, 559-583.

Clements, M.P. (2014). Forecast uncertainty - ex ante and ex post: U.S. inflation and output growth. Journal of Business and Economic Statistics 32, 206-216.

Davies, A. and K. Lahiri (1995). A new framework for analyzing survey forecasts using three-dimensional panel data. Journal of Econometrics 68, 205-227.

Draper, D. (1995). Assessment and propagation of model uncertainty. Journal of the Royal Statistical Society B 57, 45-97. 
Engelberg, J., C.F.Manski and J. Williams (2011). Assessing the temporal variation of macroeconomic forecasts by a panel of changing composition. Journal of Applied Econometrics 26, 1059-1078.

Genre, V., G. Kenny, A. Meyler and A. Timmermann (2013). Combining expert forecasts: can anything beat the simple average? International Journal of Forecasting 29, 108-121.

Geweke J. and G. Amisano (2011). Optimal prediction pools. Journal of Econometrics 164, 130-141.

Geweke J. and G. Amisano (2014). Analysis of variance for Bayesian inference. Econometric Reviews, 33, 270-288.

Giordani, P. and P. Söderlind (2003). Inflation forecast uncertainty. European Economic Review 47, 1037-1059.

Hall, P. and C.C. Heyde (1980). Martingale Limit Theory and its Application. Academic Press, New York.

Hendry, D.F. and M.P. Clements (2002). Pooling of forecasts. Econometrics Journal 5, 1-26.

Issler, J.V. and L.R. Lima (2009). A panel data approach to economic forecasting: the bias-corrected average forecast. Journal of Econometrics 152, 153-164.

Jurado, K., S.C. Ludvigson and S. Ng (2015). Measuring uncertainty. American Economic Review 105, 1177-1216.

Lahiri, K. and X. Sheng (2008). Evolution of forecast disagreement in a Bayesian learning model. Journal of Econometrics 144, 325-340.

Lahiri, K. and X. Sheng (2010). Measuring forecast uncertainty by disagreement: the missing link. Journal of Applied Econometrics 25, 514-538. 
Lahiri, K., C. Teigland and M. Zaporowski (1988). Interest rates and the subjective probability distribution of inflation forecasts. Journal of Money, Credit, and Banking 20, 233-248.

Lahiri, K., H. Peng and Y. Zhao (2015). Online learning and forecast combination in unbalanced panels. Forthcoming in Econometric Reviews.

Leamer, E.E. (1978). Specification Searches: Ad Hoc Inference with Non Experimental Data, John Wiley and Sons, Inc.

Levin, A., V. Wieland and J.C. Williams (2003). The performance of forecast-based monetary policy rules under model uncertainty. American Economic Review 93, 622-645.

Manski, C.F. (2011). Interpreting and combining heterogeneous survey forecasts. In Clements, M.P. and D.F. Hendry (eds.), Oxford Handbook of Economic Forecasting 457472. Oxford University Press.

McNees, S.K. (1992). The uses and abuses of "consensus" forecasts. Journal of Forecasting 11, 703-710.

Palm, F.C. and A. Zellner (1992). To combine or not to combine? Issues of combining forecasts. Journal of Forecasting 11, 687-701.

Pesaran, M.H. and T. Yamagata (2008). Testing slope homogeneity in large panels. Journal of Econometrics 142, 50-93.

Phillips, P.C.B. and H.R. Moon (1999). Linear regression limit theory for nonstationary panel data. Econometrica 67, 1057-1111.

Reifschneider, D. and P. Tulip (2007). Gauging the uncertainty of the economic outlook from historical forecasting errors. Federal Reserve Board, Finance and Economics Discussion Series, No. 60 . 
Sala-i-Martin, X., G. Doppelhofer and R.I. Miller (2004). Determinants of long-term growth: a Bayesian averaging of classical estimates (BACE) approach. American Economic Review 94, 813-835.

Sancetta, A. (2010). Recursive forecast combination for dependent heterogeneous data. Econometric Theory 26, 598-631.

Timmermann, A. (2006). Forecast combinations. In Elliott, G., C.W.J. Granger and A. Timmermann (eds.), Handbook of Economic Forecasting. Elsevier, 135-196.

Wallis, K.F. (2005). Combining density and interval forecasts: a modest proposal. Oxford Bulletin of Economics and Statistics 67, 983-994.

Wei, X. and Y. Yang (2012). Robust forecast combinations. Journal of Econometrics 166, 224-236.

Woodford, M. (2005). Central bank communication and policy effectiveness. Proceedings, Federal Reserve Bank of Kansas City, issue Aug, 399-474.

Yang, Y. (2004). Combining forecasting procedures: some theoretical results. Econometric Theory 20, 176-222.

Zarnowitz, V. and L.A. Lambros (1987). Consensus and uncertainty in economic prediction. Journal of Political Economy 95, 591-621. 
Table 1: Size of W test

\begin{tabular}{rrrrrrccccc}
\hline \hline & \multicolumn{9}{c}{$\sigma_{\lambda}^{2}=0.25$} & \multicolumn{3}{c}{$\sigma_{\lambda}^{2}=0.5$} & \multicolumn{3}{c}{$\sigma_{\lambda}^{2}=1$} \\
\hline \multirow{3}{*}{$\sigma_{\varepsilon}^{2}=0.05$} & & $\mathrm{n}=20$ & $\mathrm{n}=40$ & $\mathrm{n}=80$ & $\mathrm{n}=20$ & $\mathrm{n}=40$ & $\mathrm{n}=80$ & $\mathrm{n}=20$ & $\mathrm{n}=40$ & $\mathrm{n}=80$ \\
\hline \multirow{3}{*}{$\sigma_{\varepsilon}^{2}=0.5$} & $\mathrm{~T}=30$ & 0.081 & 0.071 & 0.053 & 0.084 & 0.060 & 0.054 & 0.095 & 0.061 & 0.079 \\
& $\mathrm{~T}=60$ & 0.076 & 0.077 & 0.063 & 0.075 & 0.069 & 0.056 & 0.103 & 0.094 & 0.055 \\
& $\mathrm{~T}=120$ & 0.087 & 0.077 & 0.061 & 0.078 & 0.085 & 0.072 & 0.089 & 0.063 & 0.051 \\
\hline \multirow{3}{*}{$\sigma_{\varepsilon}^{2}=1$} & $\mathrm{~T}=30$ & 0.092 & 0.060 & 0.082 & 0.099 & 0.064 & 0.062 & 0.089 & 0.076 & 0.074 \\
& $\mathrm{~T}=60$ & 0.100 & 0.081 & 0.069 & 0.102 & 0.066 & 0.057 & 0.082 & 0.060 & 0.058 \\
& $\mathrm{~T}=120$ & 0.085 & 0.101 & 0.063 & 0.084 & 0.089 & 0.067 & 0.089 & 0.075 & 0.066 \\
& $\mathrm{~T}=30$ & 0.090 & 0.075 & 0.068 & 0.091 & 0.071 & 0.075 & 0.097 & 0.090 & 0.050 \\
& $\mathrm{~T}=60$ & 0.091 & 0.074 & 0.048 & 0.092 & 0.073 & 0.058 & 0.106 & 0.062 & 0.070 \\
& $\mathrm{~T}=120$ & 0.087 & 0.078 & 0.087 & 0.097 & 0.073 & 0.058 & 0.109 & 0.071 & 0.062 \\
\hline \hline
\end{tabular}

Note: Rejection rates of $\mathrm{W}$ test under $H_{0}: \sigma_{\varepsilon i}^{2}=\sigma_{\varepsilon}^{2}$ for all $i$ at the $5 \%$ nominal level based on twosided $N(0,1)$ test and 2000 replications. The forecast errors are generated as $e_{i t}=\mu_{i t}+\lambda_{t}+\varepsilon_{i t}$, where $\mu_{i t}=c_{i} n^{-1 / 2} t^{-1 / 2}, c_{i} \sim$ i.i.d. $U(-\sqrt{3}, \sqrt{3}), \lambda_{t}=u_{t}+\theta u_{t-1}$ with $\theta=0.5, u_{t} \sim i . i . d . \sigma_{u} U(-\sqrt{3}, \sqrt{3})$, and $\varepsilon_{i t} \sim$ i.i.d. $\sigma_{\varepsilon i} U(-\sqrt{3}, \sqrt{3})$ for $i=1, \ldots, n$ and $t=1, \ldots, T$. 
Table 2: Power of $\mathrm{W}$ test

\begin{tabular}{|c|c|c|c|c|c|c|c|c|c|c|c|}
\hline & & & \multicolumn{3}{|c|}{$\sigma_{\lambda}^{2}=0.25$} & \multicolumn{3}{|c|}{$\sigma_{\lambda}^{2}=0.5$} & \multicolumn{3}{|c|}{$\sigma_{\lambda}^{2}=0.1$} \\
\hline & & & $\mathrm{n}=20$ & $\mathrm{n}=40$ & $\mathrm{n}=80$ & $\mathrm{n}=20$ & $\mathrm{n}=40$ & $\mathrm{n}=80$ & $\mathrm{n}=20$ & $\mathrm{n}=40$ & $\mathrm{n}=80$ \\
\hline \multirow{9}{*}{$\mathrm{r}=0.3$} & \multirow{3}{*}{$\sigma_{\varepsilon}^{2}=0.05$} & $\mathrm{~T}=30$ & 0.624 & 0.363 & 0.066 & 0.329 & 0.126 & 0.018 & 0.154 & 0.066 & 0.016 \\
\hline & & $\mathrm{T}=60$ & 0.956 & 0.844 & 0.163 & 0.706 & 0.365 & 0.076 & 0.395 & 0.132 & 0.004 \\
\hline & & $\mathrm{T}=120$ & 1.000 & 1.000 & 0.416 & 0.963 & 0.832 & 0.122 & 0.723 & 0.392 & 0.054 \\
\hline & \multirow{3}{*}{$\sigma_{\varepsilon}^{2}=0.5$} & $\mathrm{~T}=30$ & 0.996 & 1.000 & 0.829 & 1.000 & 0.977 & 0.594 & 0.952 & 0.847 & 0.255 \\
\hline & & $\mathrm{T}=60$ & 1.000 & 1.000 & 1.000 & 1.000 & 1.000 & 0.952 & 1.000 & 0.996 & 0.610 \\
\hline & & $\mathrm{T}=120$ & 1.000 & 1.000 & 1.000 & 1.000 & 1.000 & 1.000 & 1.000 & 1.000 & 0.982 \\
\hline & \multirow{3}{*}{$\sigma_{\varepsilon}^{2}=1$} & $\mathrm{~T}=30$ & 1.000 & 1.000 & 0.980 & 1.000 & 1.000 & 0.807 & 1.000 & 0.970 & 0.543 \\
\hline & & $\mathrm{T}=60$ & 1.000 & 1.000 & 1.000 & 1.000 & 1.000 & 1.000 & 1.000 & 1.000 & 0.953 \\
\hline & & $\mathrm{T}=120$ & 1.000 & 1.000 & 1.000 & 1.000 & 1.000 & 1.000 & 1.000 & 1.000 & 1.000 \\
\hline \multirow{9}{*}{$\mathrm{r}=0.5$} & \multirow{3}{*}{$\sigma_{\varepsilon}^{2}=0.05$} & $\mathrm{~T}=30$ & 0.685 & 0.381 & 0.239 & 0.331 & 0.108 & 0.065 & 0.120 & 0.060 & 0.009 \\
\hline & & $\mathrm{T}=60$ & 0.996 & 0.806 & 0.693 & 0.834 & 0.428 & 0.264 & 0.357 & 0.159 & 0.056 \\
\hline & & $\mathrm{T}=120$ & 1.000 & 0.996 & 0.992 & 1.000 & 0.867 & 0.712 & 0.901 & 0.420 & 0.175 \\
\hline & \multirow{3}{*}{$\sigma_{\varepsilon}^{2}=0.5$} & $\mathrm{~T}=30$ & 1.000 & 1.000 & 0.996 & 1.000 & 0.973 & 0.983 & 0.999 & 0.827 & 0.712 \\
\hline & & $\mathrm{T}=60$ & 1.000 & 1.000 & 1.000 & 1.000 & 1.000 & 1.000 & 1.000 & 0.999 & 0.996 \\
\hline & & $\mathrm{T}=120$ & 1.000 & 1.000 & 1.000 & 1.000 & 1.000 & 1.000 & 1.000 & 1.000 & 1.000 \\
\hline & \multirow{3}{*}{$\sigma_{\varepsilon}^{2}=1$} & $\mathrm{~T}=30$ & 1.000 & 1.000 & 1.000 & 1.000 & 1.000 & 1.000 & 1.000 & 0.982 & 0.976 \\
\hline & & $\mathrm{T}=60$ & 1.000 & 1.000 & 1.000 & 1.000 & 1.000 & 1.000 & 1.000 & 1.000 & 1.000 \\
\hline & & $\mathrm{T}=120$ & 1.000 & 1.000 & 1.000 & 1.000 & 1.000 & 1.000 & 1.000 & 1.000 & 1.000 \\
\hline
\end{tabular}

Note: The forecast errors are generated as $e_{i t}=\mu_{i t}+\lambda_{t}+\varepsilon_{i t}$, where $\mu_{i t}=c_{i} n^{-1 / 2} t^{-1 / 2}, c_{i} \sim$ i.i.d. $U(-\sqrt{3}, \sqrt{3}), \lambda_{t}=u_{t}+\theta u_{t-1}$ with $\theta=0.5, u_{t} \sim i . i . d . \sigma_{u} U(-\sqrt{3}, \sqrt{3})$, and $\varepsilon_{i t} \sim i . i . d . \sigma_{\varepsilon i} U(-\sqrt{3}, \sqrt{3})$ for $i=1, \ldots, n$ and $t=1, \ldots, T$. Let $R=r \times n$. Under the alternative hypothesis $\sigma_{\varepsilon i}^{2}=\sigma_{\varepsilon}^{2}$ for $i=1, \ldots,[R / 2]$ and $\sigma_{\varepsilon i}^{2} \sim \sigma_{\varepsilon}^{2} \chi^{2}(1)$ for $i=[R / 2]+1, \ldots, n$ with $[R / 2]$ being the nearest integer value of $R / 2$. The test is based on a two-sided $N(0,1)$ test and conducted at the $5 \%$ nominal level. The results are obtained based on 2000 replications. 
Table 3: Measures of historical uncertainty in inflation forecasts

\begin{tabular}{cccccc}
\hline \hline Horizon & $R M S E_{A F}$ & $R M S E_{R T}$ & $R M S E_{L P S}$ & $\mathrm{Z}$ test & $\mathrm{W}$ test \\
\hline 1 & 0.196 & 0.209 & 0.213 & $2.673^{* * *}$ & 0.365 \\
2 & 0.194 & 0.218 & 0.219 & $4.081^{* * *}$ & 0.060 \\
3 & 0.242 & 0.295 & 0.299 & $5.374^{* * *}$ & 0.127 \\
4 & 0.400 & 0.494 & 0.496 & $4.346^{* * *}$ & -0.314 \\
5 & 0.526 & 0.620 & 0.627 & $9.545^{* * *}$ & 0.790 \\
6 & 0.689 & 0.763 & 0.778 & $4.979^{* * *}$ & $1.671^{*}$ \\
7 & 0.732 & 0.882 & 0.894 & $5.636^{* * *}$ & 1.412 \\
8 & 0.779 & 0.889 & 0.897 & $6.551^{* * *}$ & 0.858 \\
\hline \hline
\end{tabular}

Note: $R M S E_{A F}$ is the conventional uncertainty measure in equation (10), $R M S E_{R T}$ is the Reifschneider and Tulip (2007)'s uncertainty measure in equation (11) and $R M S E_{L P S}$ is our suggested uncertainty measure in equation (9). Test statistic $\mathrm{Z}$ is defined in Theorem 2 in testing the null hypothesis that $R M S E_{A F}$ is the same as $R M S E_{L P S}$. Test statistic $\mathrm{W}$ is defined in Theorem 3 in testing the null hypothesis that $R M S E_{R T}$ is the same as $R M S E_{L P S}$. The inflation rate is measured as the annualaverage over annual-average percent change of GDP deflator. The actual inflation rate for 1992-2013 is taken from the first quarterly release of Federal Reserve Bank of Philadelphia "real-time" data set. The inflation forecasts used in this study are taken from the Survey of Professional Forecasters from 1991:Q1 until 2013:Q4. We construct the balanced panel by first filtering out those irregular forecasters who have responded less than $40 \%$ of time and then imputing the missing values for the regular forecasters using a simple panel regression as in Genre, et al. (2013). ${ }^{* * *},{ }^{* *}$ and $*$ indicate significance at the $1 \%, 5 \%$ and $10 \%$ level, respectively. 
Table 4: Measures of historical uncertainty in output growth forecasts

\begin{tabular}{cccccc}
\hline \hline Horizon & $R M S E_{A F}$ & $R M S E_{R T}$ & $R M S E_{L P S}$ & Z test & W test \\
\hline 1 & 0.294 & 0.297 & 0.297 & $4.966^{* * *}$ & 0.055 \\
2 & 0.374 & 0.385 & 0.385 & $5.207^{* * *}$ & 0.005 \\
3 & 0.482 & 0.523 & 0.525 & $2.854^{* * *}$ & -0.070 \\
4 & 0.788 & 0.835 & 0.839 & $4.968^{* * *}$ & 0.425 \\
5 & 1.192 & 1.246 & 1.249 & $2.612^{* * *}$ & -1.102 \\
6 & 1.448 & 1.486 & 1.490 & $4.708^{* * *}$ & $1.809^{*}$ \\
7 & 1.603 & 1.648 & 1.650 & $7.604^{* * *}$ & -0.298 \\
8 & 1.687 & 1.736 & 1.738 & $4.935^{* * *}$ & 0.210 \\
\hline \hline
\end{tabular}

Note: $R M S E_{A F}$ is the conventional uncertainty measure in equation (10), $R M S E_{R T}$ is the Reifschneider and Tulip (2007)'s uncertainty measure in equation (11) and $R M S E_{L P S}$ is our suggested uncertainty measure in equation (9). Test statistic $\mathrm{Z}$ is defined in Theorem 2 in testing the null hypothesis that $R M S E_{A F}$ is the same as $R M S E_{L P S}$. Test statistic $\mathrm{W}$ is defined in Theorem 3 in testing the null hypothesis that $R M S E_{R T}$ is the same as $R M S E_{L P S}$. The output growth is measured as the annualaverage over annual-average percent change of real GDP. The actual output growth rate for 1992-2013 is taken from the first quarterly release of Federal Reserve Bank of Philadelphia "real-time" data set. The output growth forecasts used in this study are taken from the Survey of Professional Forecasters from 1991:Q1 until 2013:Q4. We construct the balanced panel by first filtering out those irregular forecasters who have responded less than $40 \%$ of time and then imputing the missing values for the regular forecasters using a simple panel regression as in Genre, et al. (2013). ***, ** and * indicate significance at the $1 \%$, $5 \%$ and $10 \%$ level, respectively. 
Table 5: Measures of historical uncertainty from a pseudo panel of inflation forecasts

\begin{tabular}{cccccc}
\hline \hline Horizon & $R M S E_{A F}$ & $R M S E_{R T}$ & $R M S E_{L P S}$ & Z test & W test \\
\hline 1 & 0.186 & 0.194 & 0.195 & $10.576^{* * *}$ & 0.121 \\
2 & 0.218 & 0.240 & 0.244 & $17.605^{* * *}$ & 0.468 \\
3 & 0.245 & 0.312 & 0.317 & $14.644^{* * *}$ & 0.377 \\
4 & 0.428 & 0.522 & 0.529 & $20.267^{* * *}$ & 0.798 \\
5 & 0.639 & 0.722 & 0.740 & $20.122^{* * *}$ & $3.579^{* * *}$ \\
6 & 0.751 & 0.823 & 0.847 & $18.548^{* * *}$ & $5.003^{* * *}$ \\
7 & 0.763 & 0.854 & 0.863 & $15.166^{* * *}$ & $2.337^{* *}$ \\
8 & 0.762 & 0.841 & 0.851 & $16.513^{* * *}$ & $2.630^{* * *}$ \\
\hline \hline
\end{tabular}

Note: $R M S E_{A F}$ is the conventional uncertainty measure in equation (10), $R M S E_{R T}$ is the Reifschneider and Tulip (2007)'s uncertainty measure in equation (11) and $R M S E_{L P S}$ is our suggested uncertainty measure in equation (9). Test statistic $\mathrm{Z}$ is defined in Theorem 2 in testing the null hypothesis that $R M S E_{A F}$ is the same as $R M S E_{L P S}$. Test statistic $\mathrm{W}$ is defined in Theorem 3 in testing the null hypothesis that $R M S E_{R T}$ is the same as $R M S E_{L P S}$. The inflation rate is measured as the annualaverage over annual-average percent change of GDP deflator. The actual inflation rate for 1992-2013 is taken from the first quarterly release of Federal Reserve Bank of Philadelphia "real-time" data set. The inflation forecasts used in this study are taken from the Survey of Professional Forecasters from 1991:Q1 until 2013:Q4. The pseudo-balanced panel includes 21 forecasters, with each of them representing the minimum, 5 th percentile, 10 th percentile, ..., 95th percentile and the maximum of individual forecasts, respectively. ${ }^{* * *},{ }^{* *}$ and $*$ indicate significance at the $1 \%, 5 \%$ and $10 \%$ level, respectively. 
Table 6: Measures of historical uncertainty from a pseudo panel of output growth forecasts

\begin{tabular}{cccccc}
\hline \hline Horizon & $R M S E_{A F}$ & $R M S E_{R T}$ & $R M S E_{L P S}$ & $\mathrm{Z}$ test & $\mathrm{W}$ test \\
\hline 1 & 0.308 & 0.315 & 0.316 & $10.557^{* * *}$ & 0.246 \\
2 & 0.413 & 0.428 & 0.428 & $21.252^{* * *}$ & -0.103 \\
3 & 0.575 & 0.640 & 0.651 & $8.953^{* * *}$ & $2.049^{* *}$ \\
4 & 0.927 & 1.030 & 1.038 & $12.658^{* * *}$ & $2.698^{* * *}$ \\
5 & 1.288 & 1.402 & 1.409 & $12.128^{* * *}$ & $2.411^{* *}$ \\
6 & 1.414 & 1.482 & 1.483 & $15.987^{* * *}$ & $-2.856^{* * *}$ \\
7 & 1.539 & 1.632 & 1.639 & $13.666^{* * *}$ & $3.181^{* * *}$ \\
8 & 1.683 & 1.714 & 1.715 & $15.877^{* * *}$ & $-1.876^{*}$ \\
\hline \hline
\end{tabular}

Note: $R M S E_{A F}$ is the conventional uncertainty measure in equation (10), $R M S E_{R T}$ is the Reifschneider and Tulip (2007)'s uncertainty measure in equation (11) and $R M S E_{L P S}$ is our suggested uncertainty measure in equation (9). Test statistic $\mathrm{Z}$ is defined in Theorem 2 in testing the null hypothesis that $R M S E_{A F}$ is the same as $R M S E_{L P S}$. Test statistic $\mathrm{W}$ is defined in Theorem 3 in testing the null hypothesis that $R M S E_{R T}$ is the same as $R M S E_{L P S}$. The output growth is measured as the annualaverage over annual-average percent change of real GDP. The actual output growth rate for 1992-2013 is taken from the first quarterly release of Federal Reserve Bank of Philadelphia "real-time" data set. The output growth forecasts used in this study are taken from the Survey of Professional Forecasters from 1991:Q1 until 2013:Q4. The pseudo-balanced panel includes 21 forecasters, with each of them representing the minimum, 5 th percentile, 10 th percentile, ..., 95th percentile and the maximum of individual forecasts, respectively. ${ }^{* * *},{ }^{* *}$ and $*$ indicate significance at the $1 \%, 5 \%$ and $10 \%$ level, respectively. 